\title{
Topology optimization of microchannel heat sinks using a two-layer model
}

\author{
Yan, Suna; Wang, Fengwen; Hong, Jun; Sigmund, Ole
}

Published in:

International Journal of Heat and Mass Transfer

Link to article, DOI:

10.1016/j.ijheatmasstransfer.2019.118462

Publication date:

2019

Document Version

Peer reviewed version

Link back to DTU Orbit

Citation $(A P A)$ :

Yan, S., Wang, F., Hong, J., \& Sigmund, O. (2019). Topology optimization of microchannel heat sinks using a two-layer model. International Journal of Heat and Mass Transfer, 143, [118462].

https://doi.org/10.1016/j.ijheatmasstransfer.2019.118462

\section{General rights}

Copyright and moral rights for the publications made accessible in the public portal are retained by the authors and/or other copyright owners and it is a condition of accessing publications that users recognise and abide by the legal requirements associated with these rights.

- Users may download and print one copy of any publication from the public portal for the purpose of private study or research.

- You may not further distribute the material or use it for any profit-making activity or commercial gain

- You may freely distribute the URL identifying the publication in the public portal

If you believe that this document breaches copyright please contact us providing details, and we will remove access to the work immediately and investigate your claim. 


\title{
Topology optimization of microchannel heat sinks using a two-layer model
}

\author{
Suna Yan ${ }^{\mathrm{a}, *}$, Fengwen Wang ${ }^{\mathrm{b}}$, Jun Hong $^{\mathrm{a}}$, Ole Sigmund ${ }^{\mathrm{b}}$ \\ ${ }^{a}$ Key Laboratory of Education Ministry for Modern Design and Rotor-Bearing System, School of Mechanical \\ Engineering, Xi'an Jiaotong University, 710049 Xi'an, China \\ ${ }^{b}$ Department of Mechanical Engineering, Solid Mechanics, Technical University of Denmark, 2800 Kgs. Lyngby, \\ Denmark
}

\begin{abstract}
This paper investigates the topology optimization of microchannel heat sinks. A two-layer heat sink model is developed allowing to do topology optimizations at close to two-dimensional computational cost. In the model, reduced two-dimensional fluid dynamics equations proposed in the literature based on a plane flow assumption are adopted. By assuming a fourth-order polynomial temperature profile of the heat sink thermal-fluid layer and a linear temperature profile in the substrate, twodimensional heat transfer governing equations of the two layers are obtained which are thermally coupled through an out-of-plane heat flux term. Topology optimizations of a square heat sink are carried out using the two-layer model. Comparison with a three-dimensional conjugate heat transfer analysis of optimized designs in COMSOL Multiphysics validates the accuracy of the twolayer model. The re-evaluation of an optimized design by a one-layer model commonly seen in the literature shows the inadequacy of the one-layer model in predicting physical fields properly. In addition, the influence of physical and optimization parameters on the layout complexity of optimized designs is studied and related to the Peclet number. Optimizations under diffusiondominated conditions are performed and typical optimized topologies for heat conduction structures are seen.
\end{abstract}

Keywords: Microchannel heat sinks, Two-layer heat sink model, Temperature profiles, Topology optimization, Three-dimensional validation

\footnotetext{
* Corresponding author

Email address: sunayan.me@gmail.com (Suna Yan)

Part of this work was carried out while Suna Yan was a visiting student at Department of Mechanical Engineering, Technical University of Denmark.
} 


\section{Introduction}

Microchannel heat sinks have been attracting much attention since their introduction in [1] due to their high heat transfer performance. As pointed out by Tuckerman and Pease [1, microscopic channels provide very high heat transfer rate between fluid and solid phases and thereby make effective and compact cooling of, e.g., integrated electric circuits possible. Cooled by a plate-fin microchannel heat sink with optimized dimensions, the upper limit of power density of planar circuit arrays was increased nearly forty-fold compared to the suggested value at that time in [1]. Henceforth structural optimization of microchannel heat sinks has become an active research field and much work has been done.

As a simple yet effective optimization technique, sizing optimization has been widely applied to the optimization of microchannel heat sinks. Kim and Kim [2, 3] conducted analytical heat transfer analysis of plate-fin microchannel heat sinks by treating the channels and fins as a fluidsaturated porous medium. The velocity and temperature profiles were derived and the aspect ratio of channels and heat sink porosity were then optimized for minimizing the thermal resistance. Liu and Garimella 4] compared the accuracy and complexity of five heat transfer models of a plate-fin heat sink and optimized the aspect ratio and porosity using a one-dimensional resistance model. The influence of fin shape on heat transfer was investigated in [5 7. by simulating and comparing heat sinks with different fin shapes. Readers are referred to the paper 8 for an overview of sizing and configuration optimizations of microchannel heat sinks.

Different from sizing and configuration optimizations where an a priori defined structural topology is required, topology optimization has more design freedom and is well known to be able to produce unintuitive optimized designs. Topology optimization was initially developed for the stiffness optimization of mechanical structures, consisting of computing the optimal distribution of an anisotropic material [9. Density-based topology optimization approaches were developed later based on the use of material interpolations defining the relations between isotropic material parameters and the density design variables. Examples of these material laws are the SIMP (Solid Isotropic Microstructure with Penalization) model [10, 11] and RAMP (Rational Approximation of Material Properties) model [12]. Up to now, density-based approaches have been widely applied in 
the optimization of different physical problems, including heat conduction problems [13, 14, fluid problems [15, 16] and also multiphysics problems [17-19].

The design of heat sinks is a typical and challenging application of multiphysics topology optimization, where the distribution of fluid and solid is optimized by succesively solving the conjugate heat transfer problem and the optimization problem through up to hundreds of iterations. A large computational cost is therefore required. Parallel computation is essential to perform three-dimensional topology optimization of heat sinks, which has been applied to the design of natural convection heat sinks in [20, 21]. On the other hand, to circumvent solving the full threedimensional physical problems, different simplified heat transfer models have been developed in literature, especially for the topology optimization of heat sinks cooled by forced convection. A common engineering approach is to model the convection on the fluid-solid interface based on the Newton's law of cooling (NLC). The interface can be extracted by using hat functions [22, 23] or level-set expressions [24]. Topology optimizations based on the NLC models do not require the flow field solution and hence the computational cost is reduced greatly. However, in the above NLC models a single constant convection coefficient is assumed along the fluid-solid interface and the coefficient value is determined empirically. As discussed in [25], the fact that the interaction between structure and fluid keeps changing during topology optimization and the optimized designs usually have unintuitive and unanticipated layouts makes it difficult to justify the application the NLC models with empirically predetermined coefficients.

As the modeling of both solid and fluid phases becomes necessary in the topology optimization of heat sinks, some two-dimensional conjugate heat transfer models are developed without considering the influence from the third dimension. In order to predict the fluid flow and the heat transfer throughout the design domain by a single system of equations, a velocity absorption term is introduced to the momentum equation as first implemented by Borrvall and Petersson [15]. The velocity absorption term functions as a friction force applied only in the solid phase in these models. Hence, the flow model is retained in the fluid region while a vanishing flow is obtained in the solid region. Topology optimizations of microchannel heat sinks based on these two-dimensional models have been carried out in the literature. Dede [26] performed topology optimization of a multipass branching microchannel heat sink by combining the designs optimized under different boundary conditions. Matsumori et al. 27] studied the topology optimization of microchannel heat sinks subject to a constant input power for driving the flow. Topology optimizations of two-dimensional 
and three-dimensional microchannel heat sinks using level set boundary expressions were carried out in 28]. Multi-objective optimizations of heat sinks for balancing the heat transfer maximization and pressure drop minimization were studied in [29, 30.

Careful study on the pioneering work of Borrvall and Petersson [15] reveals that the velocity absorption term in effect represents the out-of-plane shear forces when reducing the three-dimensional flow to two-dimensional based on the lubrication theory where a plane flow is assumed. Therefore, the velocity absorption term never takes zero in the fluid region for planar flows, such as flows of the heat sinks as considered. The methodology has been extended to the topology optimization of Navier-Stokes channel flow problems in [16. On the other hand, if the plane flow assumption is abandoned, the generalized Stokes flow model and Navier-Stokes flow model developed in [15] and [16] respectively can still be applied to the topology optimization of three-dimensional flow problems where the flow is assumed infinitely high. In this case, the shear force meaning of the velocity absorption term is lost and the generalized models become the Brinkman-type model for porous flows. Thinking that the absorption term was derived from a seemingly unrelated flow condition for three-dimensional flows, Guest and Prévost [31] proposed a Darcy-Stokes system which is more similar to the models adopted in the above two-dimensional topology optimizations of heat sinks with a zero friction force in the fluid region.

Regarding the heat transfer process, the heat flux applied at the bottom of heat sinks is transferred to the fins and fluid through a conductive substrate which connects all the fins and forms one of the surfaces of channels, as illustrated in Figure 1 (a). Numerical simulations of plate-fin heat sinks have shown that assuming the heat flux is imposed to the fluid and solid phases evenly or to the solid phase only can result in an error of $50 \%$ and $24 \%$ respectively [4]. Therefore it is essential to take the redistribution of heat flux in the substrate and the three-dimensional heat transfer effect into account in the modeing of microchannel heat sinks. McConnell and Pingen 32 introduced a thermal substrate below the thermal-fluid layer in their heat sink model to enable heat transfer to occur in three dimensions in the topology optimization of heat sinks. This two-layer modeling approach was further developed by Haertel et al. and applied to the topology optimization of an air-cooled heat sink [33. Considering that the air-cooled heat sink has a high aspect ratio while the substrate is quite thin, the conjugate heat transfer in the thermal-fluid layer and the heat conduction in the substrate are modeled two-dimensionally, respectively. The thermal coupling between the two layers is represented through an out-of-plane heat flux term which is 
determined by a design-dependent heat transfer coefficient and the temperature difference between layers. To improve the accuracy of the two-layer model of air-cooled heat sinks, the bounds of the out-of-plane heat transfer coefficient were determined according to three-dimensional simulations and one-dimensional thermal resistance analysis of a plate-fin heat sink in 34. Van Oevelen and Baelmans [35] proposed a two-layer model by averaging the governing equations over the height of the heat sink where the velocity and temperature profiles of the fully-developed plane flow were used. However, the fluid dynamics equations before averaging have in fact been reduced to twodimensional ones with the velocity absorption term. The two-layer model was later used for the topology optimization of heat sinks in Stokes flow to study the effect of filtering techniques [36.

Inspired by the derivations of the reduced models of flow problems in [15, 16], a new twolayer model for microchannel heat sinks at low aspect ratios is developed in this paper to permit more convincing heat sink topology optimizations at close to two-dimensional computational cost. Keeping in mind that the channels of heat sinks under consideration are essentially planar, the reduced fluid dynamics equations derived in [16] where the velocity absorption term takes a finite value in the fluid phase are used but formulated in a different form. The temperature profiles in the thermal-fluid layer and substrate are derived by making suitable assumptions. By using the velocity and temperature profiles, the reduced two-dimensional heat transfer equations for the two layers are obtained which are coupled through an out-of-plane heat flux. The out-of-plane heat transfer coefficient is evaluated based on the conservation of thermal flow between layers. The accuracy of the developed two-layer heat sink model is validated by comparing with the three-dimensional simulations of optimized designs at different layout complexities in COMSOL Multiphysics.

The work in this paper is organized as follows. The two-layer model for microchannel heat sinks is developed in Section 2. Stabilized finite element formulation of the two-layer model is derived in Section 3. The overall topology optimization problem is formulated in Section 4 and implementation details are also covered. Topology optimizations of a square microchannel heat sink are performed in Section 5 and the proposed two-layer model is verified in this section. Discussion and conclusions are given in Section 6 .

\section{Two-layer heat sink model}

A sketch of a microchannel heat sink as considered in this work is shown in Figure 1 (a) which has much larger $x-y$ in-plane dimensions than its height. For the goal of deriving the two-layer 
model, the heat sink is viewed as a layered structure, consisting of the low conductive cover plate, the thermal-fluid layer and the highly conductive substrate. The cover plate is thermally insulated and together with the substrate confine the fluid flow to the thermal-fluid layer. The bottom surface of the substrate, where electrical circuits are attached, is assumed uniformly heated. The imposed heat flux is re-distributed in the substrate and transferred up to the thermal-fluid layer in the manners of conduction and convection. Fluid flowing across the thermal-fluid layer carries heat away through convection over solid fins and substrate. Under suitable assumptions, the flow velocity $\overline{\mathbf{u}}$ and temperatures in both layers $\bar{T}_{t}, \bar{T}_{b}$ have invariant profiles in the flow direction as depicted in Figure 1 (b) and explained in the following. By applying these invariant profiles, the thermal-fluid layer and substrate can be modeled two-dimensionally and heat is transferred from the substrate to the thermal-fluid layer via a virtual interface as shown in Figure 1 (c).

By doing topology optimization, the distribution of fluid and solid materials, i.e., the layout of channels and fins, in the thermal-fluid layer (design layer) is optimized to improve the heat transfer performance of the heat sink. For the analysis of heat sinks, the following assumptions are introduced: (1) the fluid constitutes a laminar and incompressible flow; (2) the fluid flow and heat transfer are in steady-state; (3) the material properties of fluid and solid phases are constant and do not change with temperature. The fluid flow is further assumed to be hydrodynamically and thermally fully developed in the derivation of the two-layer heat sink model.

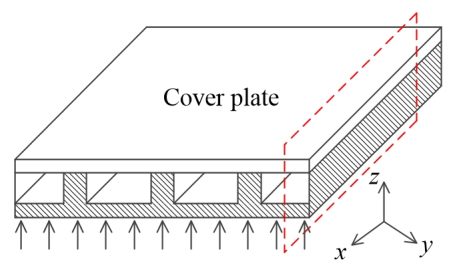

(a)

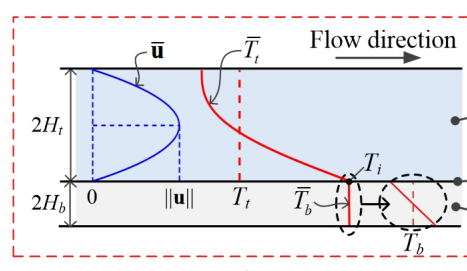

(b)

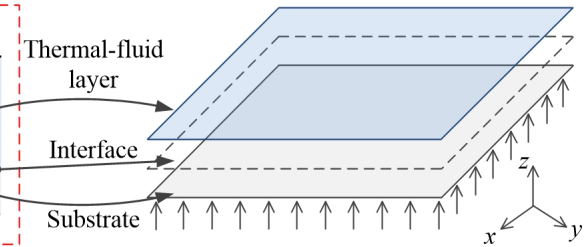

(c)

Figure 1: A microchannel heat sink as studied in this work. (a) Three-dimensional sketch, (b) Cross-section of the heat sink along the flow direction and profiles of the three-dimensional flow velocity $\overline{\mathbf{u}}$, thermal-fluid layer temperature $\bar{T}_{t}$ and substrate temperature $\bar{T}_{b}$. Here, $\|\mathbf{u}\|$ is the magnitude of the velocity vector on the mid-plane of the design layer, $T_{t}$ is the fluid bulk mean temperature, $T_{b}$ is the mean temperature of the substrate and $T_{i}$ is the temperature at the interface. (c) The two-layer heat sink model.

\subsection{Fluid dynamics modeling}

As the fluid flow is hydrodynamically developed and the design layer has much larger in-plane dimensions than its height, the flow is simplified as a Poiseuille flow between two parallel plates. 
The flow velocity profile along the height of design layer is then a parabola and does not change in the flow direction, i.e.,

$$
\begin{aligned}
& \overline{\mathbf{u}}=f_{1}(z)\left(u_{1} \mathbf{e}_{1}+u_{2} \mathbf{e}_{2}\right) \\
& f_{1}(z)=1-\left(\frac{z}{H_{t}}\right)^{2}
\end{aligned}
$$

where, $\overline{\mathbf{u}}$ is the three-dimensional velocity vector, $\mathbf{u}=\left[u_{1}, u_{2}\right]^{\mathrm{T}}$ is the velocity vector defined on the mid-plane of design layer, $\mathbf{e}_{1}$ and $\mathbf{e}_{2}$ are two of the standard basis vectors for the three-dimensional Cartesian coordinate system, $H_{t}$ is half of the height of design layer and $z \in\left[-H_{t}, H_{t}\right]$.

By using the velocity field representation in Eq. (1) and assuming uniform pressure field in $\mathrm{z}$ direction, the reduced two-dimensional continuity and momentum equations defined on the $x-y$ plane for the flow in the design layer have been derived in [15, 16, which are

$$
\begin{aligned}
& \nabla \cdot \mathbf{u}=0 \\
& \frac{6}{7} \rho_{0} \mathbf{u} \cdot \nabla \mathbf{u}=-\nabla P+\mu \nabla \cdot\left(\nabla \mathbf{u}+(\nabla \mathbf{u})^{\mathrm{T}}\right)-\frac{5 \mu}{2 H_{t}^{2}} \mathbf{u}
\end{aligned}
$$

where, $\rho_{0}$ and $\mu$ are the fluid density and dynamic viscosity, respectively and $P$ represents a rescaled in-plane pressure field. The three-dimensional velocity field and pressure field can be retained using the relations $\overline{\mathbf{u}}=f_{1}(z)[\mathbf{u}, 0]^{\mathrm{T}}$ and $\bar{P}=4 P / 5$.

The third term on the right side of the reduced momentum equation $\mathbf{F}=-\alpha \mathbf{u}$ where $\alpha=$ $5 \mu /\left(2 H_{t}^{2}\right)$ models the effect of the out-of-plane shears in fluid flow. As the material distribution in the design layer is changing during topology optimization, the reduced governing equations should be able to model not only the Poiseuille flow in the fluid region but also the vanishing flow in solid region. Hence, the inverse permeability $\alpha$ is extended to be a function of the design variable field $\gamma$, the formulation of which is detailed in Section 4.1. In the fluid region where $\gamma=1$, the factor $\alpha$ takes its smallest value and the force term $\mathbf{F}$ retains the out-of-plane shear effect, while in the solid region where $\gamma=0$, a very large $\alpha$ is attained to penalize the flow and obtain a vanishing flow velocity.

\subsection{Heat transfer modeling}

Similar to the reduction of fluid dynamics equations, the heat transfer equations can also be reduced to two-dimensional by using the temperature profile formulations of the heat sinks. On the 
other hand, the heat transfer process involves both the thermal-fluid layer and substrate. Hence the thermal coupling between the two layers is considered after the investigation of each layer to couple the reduced governing equations to one system. Specifically, a fourth-order polynomial temperature profile and a linear temperature profile are derived for the thermal-fluid layer and substrate respectively as depicted in Figure 1 (b). Substituting the temperature profiles and the velocity profile into the weak formulations of the heat transfer equations and then integrating, the dependency of the formulations on $z$ dimension can be eliminated and some rescaling constants are introduced. By localizing the resulting two-dimensional weak formulations, reduced and rescaled heat transfer governing equations of the two layers are then obtained. In the following, the derived temperature profiles and reduced heat transfer governing equations are given directly for simplicity. Detailed derivations can be found in Appendix A.

Considering that the fluid flow is hydrodynamically and thermally developed and assuming the heat flux applied at the bottom of flow is constant, then the profile of the non-dimensional temperature variable defined below is invariant in the flow direction [37.

$$
\begin{aligned}
& \theta_{t}=\frac{T_{i}-\bar{T}_{t}}{T_{i}-T_{t}}=f_{2}(z) \\
& f_{2}(z)=\frac{35}{416}\left[\left(\frac{z}{H_{t}}\right)^{4}-6\left(\frac{z}{H_{t}}\right)^{2}+8\left(\frac{z}{H_{t}}\right)+13\right]
\end{aligned}
$$

where, $\bar{T}_{t}$ represents the three-dimensional temperature field of design layer, $T_{t}=\int_{-H_{t}}^{H_{t}}\|\overline{\mathbf{u}}\| \bar{T}_{t} \mathrm{~d} z /\left(2 H_{t} u_{m}\right)$ is the bulk mean temperature of the fluid, $u_{m}=\int_{-H_{t}}^{H_{t}}\|\overline{\mathbf{u}}\| \mathrm{d} z /\left(2 H_{t}\right)$ is the mean velocity of the flow, $T_{i}$ is the temperature at the interface between thermal-fluid layer and substrate and $z \in\left[-H_{t}, H_{t}\right]$.

The two-dimensional heat transfer governing equation for the thermal-fluid design layer is derived based on the temperature profile Eq. (3) as

$$
\frac{2}{3} \rho_{0} C\left(\mathbf{u} \cdot \nabla T_{t}\right)-\frac{49}{52} \nabla \cdot\left(k_{t} \nabla T_{t}\right)-\frac{1}{2 H_{t}} \cdot h_{t}\left(T_{i}-T_{t}\right)=0
$$

where, $C$ is the heat capacity of fluid, $k_{t}=k_{t}(\gamma)$ is the effective thermal conductivity and its formulation is defined in Section 4.1 which equals the fluid conductivity $k_{f}$ in fluid region and that of solid material $k_{s}$ in fins. The fluid bulk mean temperature $T_{t}$ is the state variable in this reduced two-dimensional governing equation. $q_{i}^{\prime \prime}=h_{t}\left(T_{i}-T_{t}\right)$ with $h_{t}=35 k_{t} /\left(26 H_{t}\right)$ is an out-of-plane 
heat flux which shows the thermal coupling between thermal-fluid layer and substrate.

The three-dimensional heat conduction governing equation for the substrate is reduced based on the following non-dimensional temperature variable whose profile is invariant in $x / y$ directions.

$$
\begin{aligned}
& \theta_{b}=\frac{T_{i}-\bar{T}_{b}}{T_{i}-T_{b}}=f_{3}(z) \\
& f_{3}(z)=1-\frac{z}{H_{b}}
\end{aligned}
$$

where, $\bar{T}_{b}$ represents the three-dimensional temperature field of substrate, $T_{b}=\int_{-H_{b}}^{H_{b}} \bar{T}_{b} \mathrm{~d} z /\left(2 H_{b}\right)$ is the mean temperature and equals the temperature at the mid-plane of substrate for the linear temperature profile used here, $H_{b}$ is half of the height of the substrate and $z \in\left[-H_{b}, H_{b}\right]$.

The two-dimensional heat conduction governing equation for the substrate is derived as

$$
-\frac{k_{b}}{2} \nabla^{2} T_{b}+\frac{1}{2 H_{b}} \cdot h_{b}\left(T_{b}-T_{i}\right)-\frac{q_{0}^{\prime \prime}}{2 H_{b}}=0
$$

where, $k_{b}=k_{s}$ is the thermal conductivity of the solid material, $q_{0}^{\prime \prime}$ is the heat flux applied on the bottom surface of the substrate. The mean temperature $T_{b}$ is the state variable in the reduced governing equation. The out-of-plane heat flux $q_{i}^{\prime \prime}=h_{b}\left(T_{b}-T_{i}\right)$ with $h_{b}=k_{b} / H_{b}$ also represents the heat transfer from substrate to thermal-fluid layer.

Using the concept of thermal circuits, the heat flux representing the thermal coupling between layers can also be expressed as $q_{i}^{\prime \prime}=h\left(T_{b}-T_{t}\right)$, where $h=h_{t} h_{b} /\left(h_{t}+h_{b}\right)$. As the effective thermal conductivity of the thermal-fluid layer $k_{t}$ is design-dependent and equals that of the fluid and solid materials at corresponding regions, the general heat transfer coefficient $h$ is much higher in fins than in fluid regions. The out-of-plane heat flux $q_{i}^{\prime \prime}$ can thus flexibly represent the conductive and convective heat transfer between the thermal-fluid layer and substrate. The system of reduced two-dimensional governing equations for the heat transfer in the heat sink are

$$
\begin{aligned}
& \frac{2}{3} \rho_{0} C\left(\mathbf{u} \cdot \nabla T_{t}\right)-\frac{49}{52} \nabla \cdot\left(k_{t} \nabla T_{t}\right)-\frac{h}{2 H_{t}}\left(T_{b}-T_{t}\right)=0 \\
& -\frac{k_{b}}{2} \nabla^{2} T_{b}+\frac{h}{2 H_{b}}\left(T_{b}-T_{t}\right)-\frac{q_{0}^{\prime \prime}}{2 H_{b}}=0
\end{aligned}
$$

In summary, the governing equations of the heat transfer process of the heat sinks under consideration are Eqs. (2) and (7), respectively. The system is weakly coupled in the sense that the 
flow field is independent of the temperature field, whereas the temperature field is influenced by the flow field due to the convection effect. The dependency of material inverse permeability $\alpha$, effective thermal conductivity $k_{t}$ and heat transfer coefficient $h$ on the design variable field $\gamma$ enables the description of flow regions and solid regions using the same set of equations.

\section{Finite element formulation}

The computational domain consisting of thermal-fluid design layer and substrate is discretized by 4 -node quadrilateral finite elements. When the standard Galerkin finite element formulation is used, two main sources of numerical instabilities may occur. One is oscillations in the pressure field caused by inappropriate pairs of velocity and pressure interpolations which violate the Ladyzhenskaya-Babuska-Brezzi (LBB) stability condition. The other is the occurrence of node-tonode oscillations, 'wiggles', in velocity field and temperature field emanating from the steep solution gradient especially in convection dominated problems. Therefore, the pressure-stabilizing/PetrovGalerkin (PSPG) term [38] and streamline-upwind/Petrov-Galerkin (SUPG) term [39] are added to the weak form of governing equations to construct stabilized finite element formulation. The PSPG term helps circumvent the LBB condition and hence the computationally desirable equal-order velocity and pressure interpolations become viable. The SUPG term solves the wiggle problem by adding a proper amount of diffusion in the flow direction which can also be viewed as a streamline upwind perturbation to the weighting functions.

The stabilized weak forms for the continuity and momentum equations given by Eq. (2) are

$$
\begin{gathered}
\int_{\Omega_{t}} \phi^{h} \frac{\partial u_{i}^{h}}{\partial x_{i}} d \Omega+\sum_{e=1}^{n_{e l}} \int_{\Omega_{t}^{e}} \frac{\tau_{\mathrm{PS}}}{\left(6 \rho_{0} / 7\right)} \frac{\partial \phi^{h}}{\partial x_{i}} \mathcal{R}_{i}^{u}\left(\mathbf{u}^{h}, P^{h}\right) d \Omega=0 \\
\int_{\Omega_{t}} \frac{6}{7} \rho_{0} \omega_{i}^{h}\left(u_{j}^{h} \frac{\partial u_{i}^{h}}{\partial x_{j}}\right) d \Omega+\int_{\Omega_{t}} \frac{\partial \omega_{i}^{h}}{\partial x_{j}}\left[-P \delta_{i j}+\mu\left(\frac{\partial u_{i}}{\partial x_{j}}+\frac{\partial u_{j}}{\partial x_{i}}\right)\right] d \Omega \\
+\int_{\Omega_{t}} \alpha \omega_{i}^{h} u_{i}^{h} d \Omega-\int_{\Gamma_{h}} \omega_{i}^{h} \mathcal{T}_{i} d \Gamma+\sum_{e=1}^{n_{e l}} \int_{\Omega_{t}^{e}} \tau_{\mathrm{SU}}\left(u_{j}^{h} \frac{\partial \omega_{i}^{h}}{\partial x_{j}}\right) \mathcal{R}_{i}^{u}\left(\mathbf{u}^{h}, P^{h}\right) d \Omega=0
\end{gathered}
$$

where, $\Omega_{t}=\sum_{e=1}^{n_{e l}} \Omega_{t}^{e}$ represents the design layer, $\mathbf{u}^{h}=\left[u_{1}^{h}, u_{2}^{h}\right]^{\mathrm{T}}$ and $P^{h}$ are the approximated velocity field and pressure field respectively, $\boldsymbol{\omega}^{h}=\left[\omega_{1}^{h}, \omega_{2}^{h}\right]^{\mathrm{T}}$ and $\phi^{h}$ are the test functions, $\mathcal{T}_{i}$ is the 
boundary traction, $\mathcal{R}_{i}^{u}\left(\mathbf{u}^{h}, P^{h}\right)$ is the residual form of the momentum equation, $\tau_{\mathrm{PS}}$ is the PSPG stabilization parameter, $\tau_{\mathrm{SU}}$ is the SUPG stabilization parameter for the momentum equation.

The stabilized formulation for the convection-diffusion equation in design layer and the standard Galerkin formulation for the heat conduction equation in the substrate are

$$
\begin{gathered}
\int_{\Omega_{t}} \frac{2}{3} \rho_{0} C \varphi_{t}^{h}\left(u_{j}^{h} \frac{\partial T_{t}^{h}}{\partial x_{j}}\right) d \Omega+\int_{\Omega_{t}} \frac{49}{52} k_{t} \frac{\partial \varphi_{t}^{h}}{\partial x_{j}} \frac{\partial T_{t}^{h}}{\partial x_{j}} d \Omega-\int_{\Omega_{t}} \frac{h}{2 H_{t}} \varphi_{t}^{h}\left(T_{b}^{h}-T_{t}^{h}\right) d \Omega \\
-\int_{\Gamma_{t}^{q}} \varphi_{t}^{h} q_{t}^{\prime \prime} d \Gamma+\sum_{e=1}^{n_{e l}} \int_{\Omega_{t}^{e}} \tau_{\mathrm{SU}_{\mathrm{T}}}\left(u_{j}^{h} \frac{\partial \varphi_{t}^{h}}{\partial x_{j}}\right) \mathcal{R}_{T}\left(\mathbf{u}^{h}, T_{t}^{h}, T_{b}^{h}\right) d \Omega=0 \\
\int_{\Omega_{b}} \frac{k_{b}}{2} \frac{\partial \varphi_{b}^{h}}{\partial x_{j}} \frac{\partial T_{b}^{h}}{\partial x_{j}} d \Omega+\int_{\Omega_{b}} \frac{h}{2 H_{b}} \varphi_{b}^{h}\left(T_{b}^{h}-T_{t}^{h}\right) d \Omega-\int_{\Omega_{b}} \varphi_{b}^{h} d \Omega \frac{q_{0}^{\prime \prime}}{2 H_{b}}-\int_{\Gamma_{b}^{q}} \varphi_{b}^{h} q_{b}^{\prime \prime} d \Gamma=0
\end{gathered}
$$

where, $\Omega_{b}$ denotes the substrate domain, $T_{t}^{h}$ and $T_{b}^{h}$ are the approximated temperature fields of the design layer and substrate respectively, $\varphi_{t}^{h}$ and $\varphi_{b}^{h}$ are test functions, $q_{t}^{\prime \prime}$ and $q_{b}^{\prime \prime}$ are boundary

heat fluxes, $\mathcal{R}_{T}\left(\mathbf{u}^{h}, T_{t}^{h}, T_{t}^{b}\right)$ is the residual form of the convection-diffusion equation and $\tau_{\mathrm{SU}_{\mathrm{T}}}$ is the corresponding SUPG stabilization parameter.

The stabilization parameters play an important role in the stabilized finite element method and their values are usually a function of local length scales and velocity scales. Here, the formulations of stabilization parameters proposed in [40] and extended in [25] are used, where the local length scales are decided based on the local solution gradients and the local material inverse permeabilities are also taken into account in computing the parameters for the flow equations. Hence, the flow equations are non-linear with respect to the velocity field due to the convection term and also the stabilization parameters $\tau_{\mathrm{SU}}$ and $\tau_{\mathrm{PS}}$. The thermal equations are implicitly non-linear with respect to the temperature field due to the stabilization parameter $\tau_{\mathrm{SU}_{\mathrm{T}}}$.

\section{Topology optimization problem}

\subsection{Material interpolation schemes}

In topology optimization the design of structures is represented by material distribution, i.e., the design variable field which is denoted here as $\gamma$. Specifically for the design of the heat sinks under consideration, the material at each point $x$ in design domain is either fluid with $\gamma(x)=1$ or solid with $\gamma(x)=0$. The topology optimization of heat sinks usually requires at least several 
hundreds of iterations to obtain a good design. In addition, the governing equations (8) to (11) are non-linear and should be solved through an iterative procedure. Mathematically well-founded and efficient gradient-based optimization algorithms therefore should be used, which require the design variables to be continuous in the range of $[0,1]$. The physical meaning of design variables can then be understood as the volume fraction occupied by fluid at each point. RAMP-style interpolations [12, 15] are used to define the material property-to-design variable relations. The inverse permeability $\alpha$ is formulated as

$$
\alpha(\gamma)=\alpha_{s}+\left(\alpha_{f}-\alpha_{s}\right) \gamma \frac{1+q_{\alpha}}{\gamma+q_{\alpha}}
$$

where, $\alpha_{f}$ and $\alpha_{s}$ are the inverse permeabilities of fluid and solid regions respectively and $q_{\alpha}$ is a penalization parameter which is used to suppress intermediate values of design variables, since the final goal of topology optimization is to obtain discrete-valued designs.

As discussed in Section 2.1, the force term $\mathbf{F}=-\alpha \mathbf{u}$ should retain the out-of-plane shear stress in the fluid region, thus $\alpha_{f}=5 \mu /\left(2 H_{t}^{2}\right)$ is used and $H_{t}$ is half of the channel height. As for the solid region, a zero channel height should be used in theory. But for implementation purposes, a very small channel height $H_{t} / 100$ is used instead and thus $\alpha_{s}=5 \mu /\left[2\left(H_{t} / 100\right)^{2}\right]$. The same ratio between $\alpha_{s}$ and $\alpha_{f}$ is also used in [16.

The effective thermal conductivity $k_{t}$ is interpolated as

$$
k_{t}(\gamma)=k_{f}+\left(k_{s}-k_{f}\right) \frac{1-\gamma}{1+q_{k} \gamma}
$$

where, $k_{f}$ and $k_{s}$ are the thermal conductivities of fluid and solid respectively and $q_{k}$ is a penalization parameter to suppress intermediate design variables through the effect on material thermal conductivity. Based on the interpolation of the effective thermal conductivity, the relation of heat transfer coefficient between layers and design variable $h=h(\gamma)$ can be readily obtained.

In density-based topology optimization approaches, the design variable field is also discretized by the finite element mesh and each element is assigned with a unique design variable. Hence, the design of the heat sink is represented by the design variable vector $\gamma$. As the material inverse permeability and effective thermal conductivity are interpolated as functions only depending on the design variable field, they are also represented by the corresponding vectors and can be substituted in the stabilized formulations $80 \sim(11)$. 


\subsection{Problem formulation}

The heat transfer performance of a heat sink can be measured by its thermal resistance, which is defined as

$$
R=\frac{T_{\max }-T_{0}}{q_{0}^{\prime \prime} A}
$$

where, $q_{0}^{\prime \prime}$ is the heat flux imposed by the planar electric circuits which are attached to the substrate and have an area of $A, q_{0}^{\prime \prime} A$ is then the power to be dissipated by the heat sink, $T_{0}$ is the input coolant temperature, $T_{\max }$ is the maximum temperature of the heat sink which is expected to appear in the substrate. As $q_{0}^{\prime \prime} A$ and $T_{0}$ are usually fixed, the maximum temperature of the substrate is selected as the objective function in the topology optimization of heat sinks in this work. The topology optimization problem is then formulated as

$$
\begin{array}{ll}
\min _{\boldsymbol{\gamma}}: & T_{b, \max } \\
\text { s.t. : } & \text { equillibrium equations } 8 \sim 11, \\
& \sum_{e=1}^{n_{d}} \gamma_{e} A_{e} \leqslant f A_{\Omega_{d}}, \\
& g_{i}(\boldsymbol{\gamma}) \leqslant 0, \quad i=1, \ldots, m \\
& \mathbf{0} \leqslant \boldsymbol{\gamma} \leqslant \mathbf{1} .
\end{array}
$$

where, $T_{b, \max }$ is the maximum temperature of the substrate, $A_{\Omega_{d}}$ is the area of design domain $\Omega_{d}$ which is a subset of the design layer and discretized into $n_{d}$ elements, $\gamma_{e}$ is the design variable of element $e$ and $A_{e}$ is the elemental area, the first inequality hence constrains the volume fraction of fluid to be no larger than $f$ in the design domain. Whether or not other inequality constraints $g_{i}(\gamma)$ are needed depends on the problems under consideration and the requirements of designers. An example is when the inflow velocity distribution is prescribed, an additional pressure drop constraint may be applied to limit the power necessary to drive flow through the heat sink. Considering that the max-operator is non-differentiate, $T_{b, \max }$ is approximated by the generalized $p$-norm of the substrate nodal temperatures as

$$
T_{b, \max } \approx\left(\frac{1}{N} \sum_{i=1}^{N} T_{b, i}^{p}\right)^{1 / p}
$$


where, $N$ is the total number of substrate nodes and $T_{b, i}$ is the temperature of node $i$. The value of the temperature norm parameter $p$ should be able to capture the trend of maximum temperature in the optimization process and also ensure adequate smoothness of the objective function. Based on the studies in [13, 41, 42] on the effect of the norm parameter, $p=10$ is used in our work.

The volume constraint in topology optimization problems is usually used for the consideration of material cost or structural weight to avoid optimized designs made of the better material completely. In the design of forced-convection heat sinks, however, a trade-off between increasing the convection surface and reducing the flow resistance is expected in the optimized designs. Therefore, optimized heat sinks should not be blank without any fins or solid blocks without any channels even if no volume constraint is applied, when appropriate boundary conditions are applied, e.g., the allowable pressure drop is large enough. On the other hand, the volume constraint also works together with the penalized material interpolation schemes to suppress intermediate design variables and it avoids congestion in the design domain. For heat sinks design, the increase in flow resistance also helps suppress intermediate design variables to some extent. A trivial volume constraint is thus used in most cases of this work by setting $f=1$ unless otherwise stated.

\subsection{Implementations}

To avoid checkerboards in and mesh dependency of design results, the density filtering technique [43, 44] is applied. The element-wise design variables are replaced by the weighted average of design variables of their neighbours within a mesh-independent region defined by the filter radius $r_{\text {min }}$. The filtered design variables are the ones with physical meaning and used to compute material properties by Eqs. 12 and 13 .

The distribution of fluid and solid is optimized by using the Method of Moving Asymptotes (MMA) [45] as the optimization algorithm. Sensitivities of the objective function and volume constraint with respect to the design variables are computed based on the adjoint approach. As in [25], the dependency of stabilization parameters on velocity and temperature fields is neglected in computing the sensitivities. The accuracy of these approximate sensitivities are validated through finite difference checks.

The optimization procedure is considered converged when the maximum change of design variables from iteration to iteration is smaller than a certain value ( 0.01 in this work). In addition, the optimization is allowed to run a maximum of 250 iterations. To end up with better optimized 
designs, a continuation approach to gradually increase the penalization parameter(s) is adopted. Specifically, the penalization on effective conductivity $q_{k}$ is updated in a sequence of $[1,3,10,30]$ every 50 iterations or upon convergence, while the penalization parameter on inverse permeability is fixed at $q_{\alpha}=0.1$. After optimization, the results are projected to obtain strictly black-white designs by a simple threshold projection satisfying the volume ratio of fluid and solid phases.

\section{Case study}

Topology optimization of a square heat sink is performed in this section using the proposed two-layer model and methodology. The heat sink is sketched in Figure 2 where a square substrate is cooled by a thermal-fluid design layer which has a square flow domain and additional inlet and outlet regions. Normal flow is specified at inlet and outlet boundaries and other boundaries of the design layer satisfy the no-slip boundary condition. The pressure drop of the heat sink is prescribed and imposed by setting $\mathcal{T}_{1}=\Delta \mathrm{P}$ and $\mathcal{T}_{1}=0$ at the inlet and outlet boundaries, respectively, where $\mathcal{T}_{1}$ denotes the boundary traction in the $x$-direction. A constant heat flux $q_{0}^{\prime \prime}=6 \times 10^{4} \mathrm{~W} / \mathrm{m}^{2}$ is applied over the bottom of the substrate. The inlet coolant temperature is fixed at $T_{0}=0^{\circ} \mathrm{C}$, while other boundaries of the heat sink are thermally insulated. Water is used as the coolant and silicon is the material of the substrate and fins. Material properties and dimensions of the heat sink are listed in Table 1. As the change of material properties with temperature is not considered in the following case studies, the problem is linear with respect to the heat flux $q_{0}^{\prime \prime}$ and scaling of the heat flux will not change the design results.

Considering that the heat sink and the loading and boundary conditions are symmetric with respect to the horizontal central line, only the upper half of the heat sink is considered in topology optimization and a symmetric boundary condition is applied on the central line. The half of the square region in the design layer is discretized into 440-by-220 elements and the same for the substrate. The inlet and outlet regions are discretized into 110-by-55 elements, respectively. A filter radius of $r_{\text {min }}=L / 100$ is used in the density filtering, where $L$ is the side length of the square flow region. Two different settings of design domain are used, as illustrated in Figure 2 In the first setting, only the square flow region is designable and the inlet and outlet are specified to be fluid, while in the second one the outlet and part of the inlet away from the inflow boundary are also allowed to be optimized. The initial guess for topology optimizations is $\gamma=0.9$ in the square flow region and $\gamma=1$ in inlet and outlet regions. 

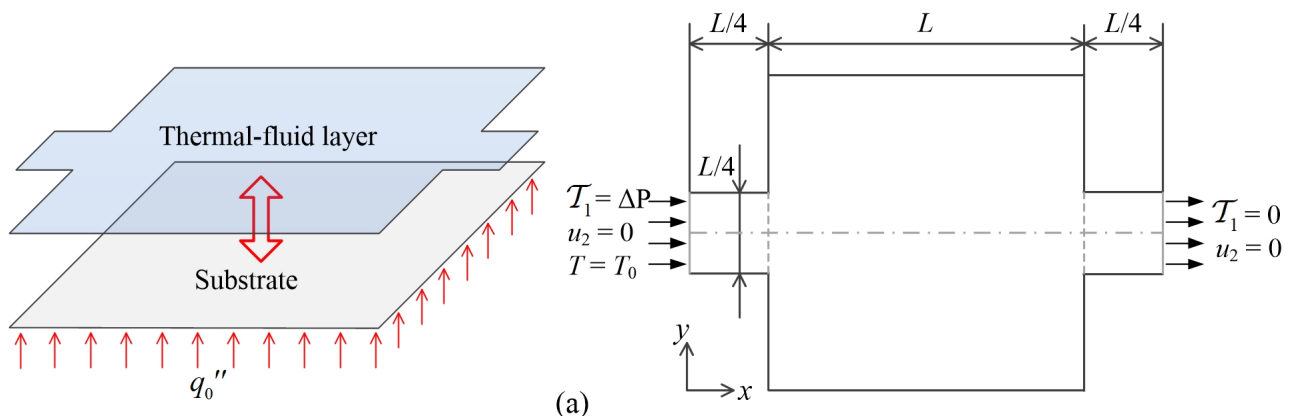

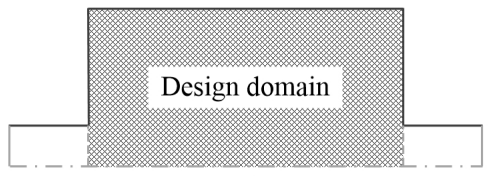

(b)

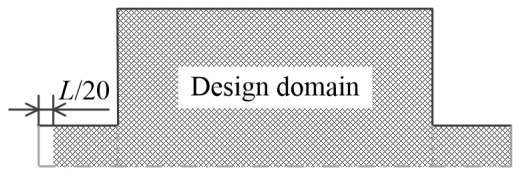

(c)

Figure 2: The square heat sink for topology optimization is sketched in (a). The two different design domain settings are illustrated in (b) and (c).

Table 1: Material properties and dimensions of the heat sink for topology optimization.

\begin{tabular}{llll}
\hline Parameters & Symbols & Values & $\mathrm{Units}$ \\
\hline Water density & $\rho_{0}$ & 998 & $\mathrm{~kg} / \mathrm{m}^{3}$ \\
Water viscosity & $\mu$ & $1.004 \times 10^{-3}$ & $\mathrm{~Pa} \cdot \mathrm{s}$ \\
Water heat capacity & $C$ & 4180 & $\mathrm{~J} /(\mathrm{kg} \cdot \mathrm{K})$ \\
Water conductivity & $k_{f}$ & 0.598 & $\mathrm{~W} /(\mathrm{m} \cdot \mathrm{K})$ \\
Silicon conductivity & $k_{s}$ & 149 & $\mathrm{~W} /(\mathrm{m} \cdot \mathrm{K})$ \\
Length of heat sink & $L$ & 10 & $\mathrm{~mm}$ \\
Height of design layer & $2 H_{t}$ & 0.5 & $\mathrm{~mm}$ \\
Height of substrate & $2 H_{b}$ & 0.2 & $\mathrm{~mm}$ \\
\hline
\end{tabular}




\subsection{Optimized designs}

Topology optimizations of the heat sink have been done using different pressure drop values $(\Delta \mathrm{P}$ $=10 \mathrm{~Pa}, 50 \mathrm{~Pa}, 200 \mathrm{~Pa}$ ), flow models (Navier-Stokes model and Stokes model) and design domain settings. The optimized designs are shown in Figure 3. Only a few discrete fins exist in the designs for a pressure drop of $10 \mathrm{~Pa}$. More pin fins in streamlined shapes show up in the optimized designs with increase in prescribed pressure drop and in the designs for $\Delta \mathrm{P}=200 \mathrm{~Pa}$ the distributions of fins form complex channel networks. The change of fin layouts with pressure drop shows a balance between increasing convection surface and reducing flow resistance in the optimized designs.

The designs under a pressure drop of $10 \mathrm{~Pa}$ are quite similar apart from the one using NavierStokes flow model and designable inlet and outlet setting (NSD setting). Comparison of the velocity fields of the similar designs shows that the inertia term in the Navier-Stokes equation produces slight differences mainly in the inlet and outlet regions, while the temperature fields of these designs are very similar. In contrast, the designs of different flow models present different layouts under pressure drops of $50 \mathrm{~Pa}$ and $200 \mathrm{~Pa}$. Thick branches of channels are observed close to the outlet in the NavierStokes designs. Inclined inlet or large and extending round corners beside the inlet are formed in the Navier-Stokes designs under a pressure drop of $200 \mathrm{~Pa}$ to bend the flow smoothly.

Optimization histories of designs under a pressure drop of $200 \mathrm{~Pa}$ are given in Figure 4 The maximum temperatures follow similar trends to those of the corresponding objective functions during optimization. Each time the penalization parameter on material effective thermal conductivity is updated, the optimizations show slight oscillations at the beginning and then become stable gradually. The designs in Figure 3 are re-evaluated under pressure drops different from the design value and the cross-check results are plotted in Figure 5. As expected, each design performs best under the pressure drop for which it is optimized, and thereby the effectiveness of topology optimization is justified.

\subsection{Model validation}

To validate the developed two-layer heat sink model, all the optimized designs in Figure 3 are built as full three-dimensional heat sinks and re-evaluated using the conjugate heat transfer module

in Comsol Multiphysics software. As an example, the designs optimized using the NSP setting and the corresponding three-dimensional heat sinks are shown in Figure 6. The three-dimensional heat sinks are meshed using body-fitted grids and three-dimensional physical fields are resolved. 


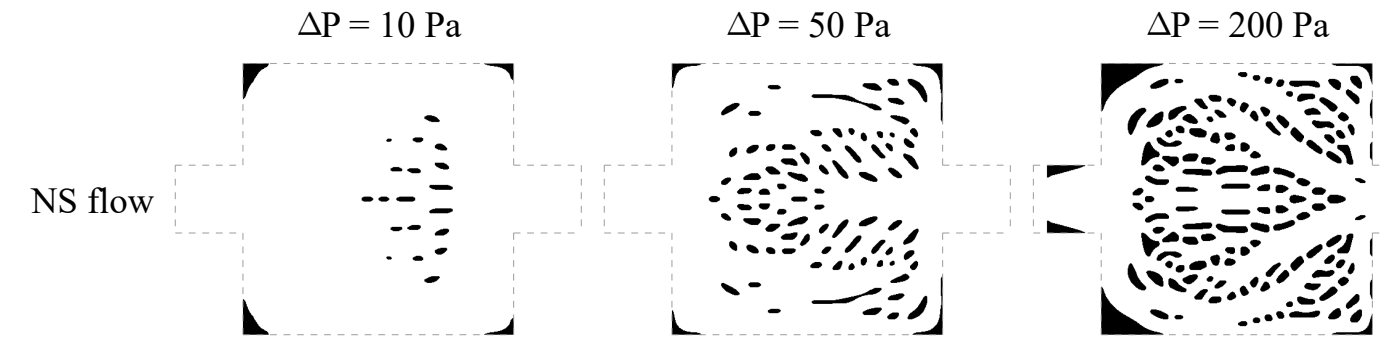

(a)
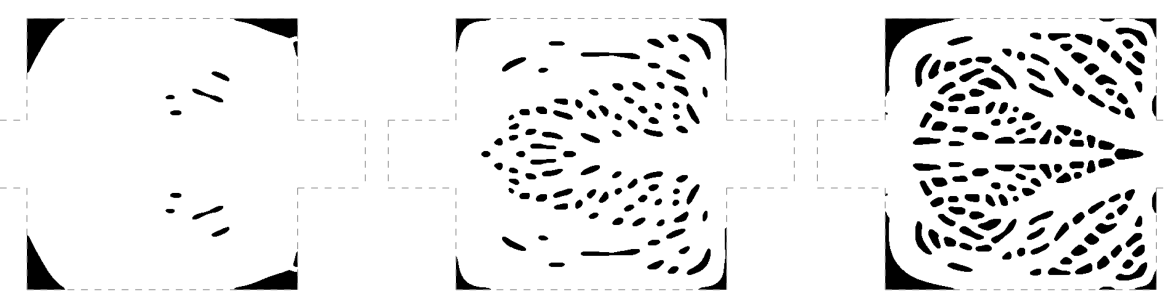

(b)
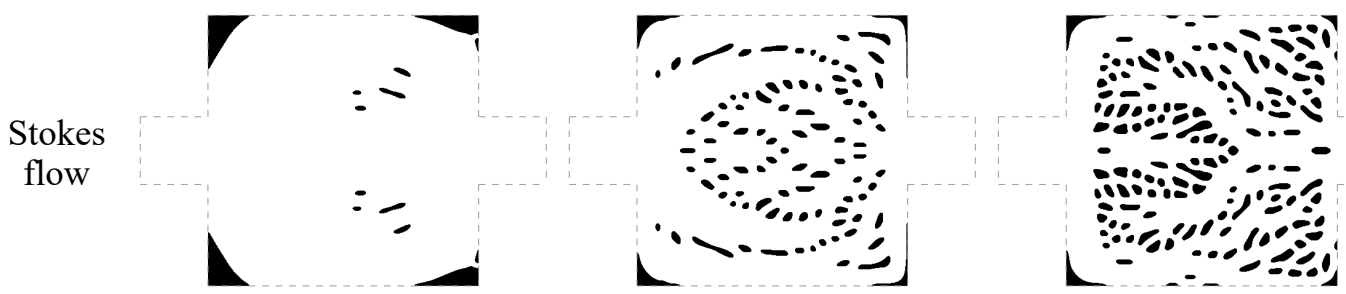

(c)
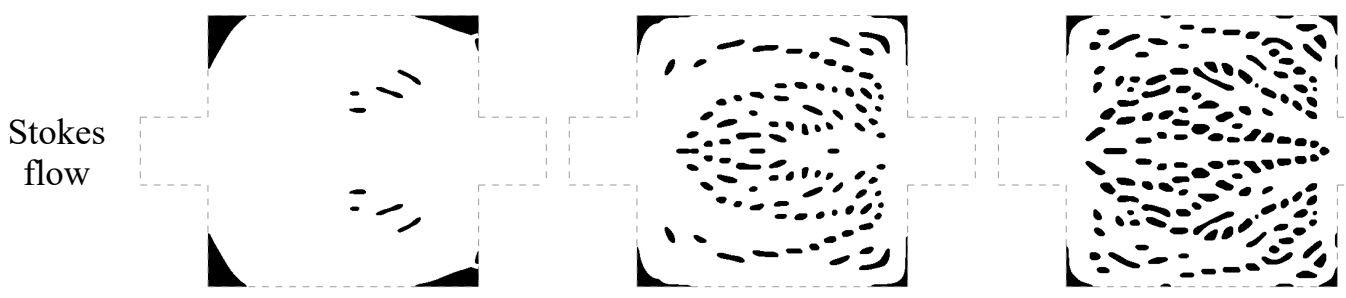

(d)

Figure 3: Optimized designs under pressure drops of $10 \mathrm{~Pa}$ (left), $50 \mathrm{~Pa}$ (middle), $200 \mathrm{~Pa}$ (right). Specifically, (a) are designs of Navier-Stokes model and designable inlet and outlet setting (NSD setting), (b) are of Navier-Stokes model and passive inlet and outlet setting (NSP setting), (c) are of Stokes model and designable inlet and outlet setting

(SD setting), (d) are of Stokes model and passive inlet and outlet setting (SP setting). (Black regions are fins and white regions are channels) 


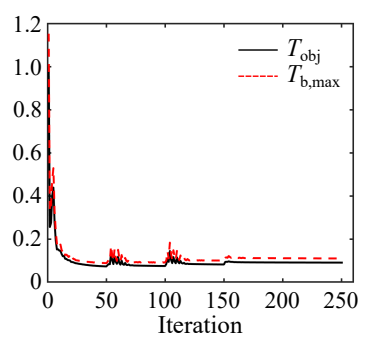

(a)

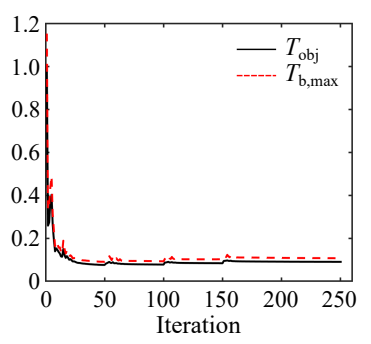

(b)

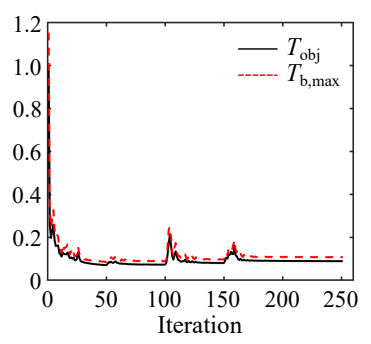

(c)

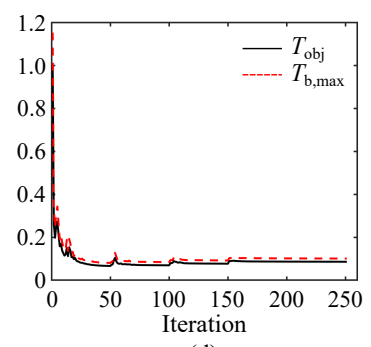

(d)

Figure 4: Optimization histories of the objective function $T_{\mathrm{obj}}$ and maximum substrate temperature $T_{\mathrm{b}, \max }$ of designs under a pressure drop of $200 \mathrm{~Pa}$. Objectives and maximum temperatures scaled by objectives of the correpsonding initial guesses are plotted. The sub-figures (a)-(d) are for the corresponding designs in Figure 3 (a)-(d), respectively.

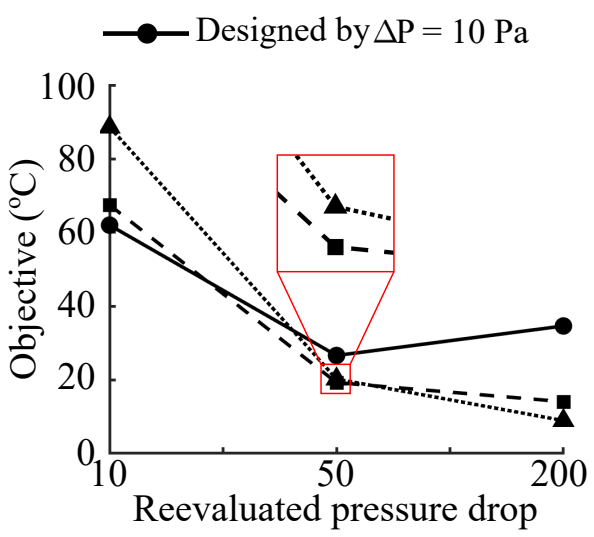

(a)

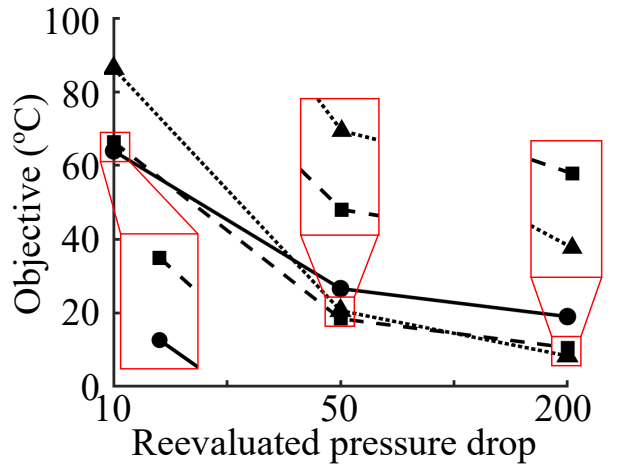

(c)

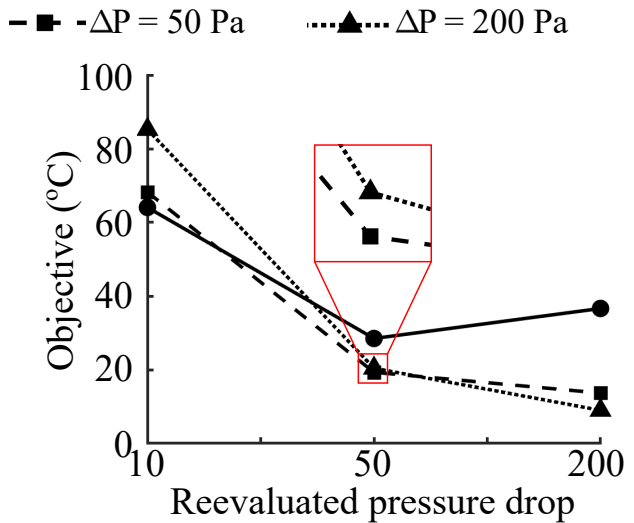

(b)

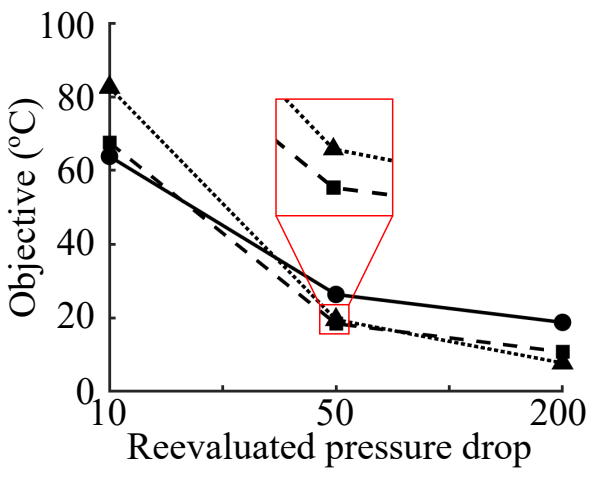

(d)

Figure 5: Cross-check of designs in Figure 3 The sub-figures (a)-(d) are for the corresponding designs in Figure 3 (a)-(d), respectively. 
Two-dimensional slice plots of velocity and temperature fields are taken to compare with the twolayer model results. Figure 7 (a)-(c) show the physical fields of the designs optimized for pressure drops of $10 \mathrm{~Pa}, 50 \mathrm{~Pa}$ and $200 \mathrm{~Pa}$ respectively. The upper three sub-figures in Figure 7 (a)-(c) are respectively the velocity magnitude field (left) and temperature field (middle) on the mid-plane of the design layer and temperature field on the mid-plane of the substrate (right) computed by the two-layer heat sink model. The lower sub-figures are the fields from the three-dimensional simulations. Different from the two-layer model where fluid and solid domains are governed by the same flow equations and distinguished from each other through the force term, the two phases are separately modeled in the three-dimensional re-evaluations and thus the fins are shown as blank regions in the corresponding velocity plots. It can be seen that the velocity fields computed by the two-layer model and three-dimensional simulations are in good agreement for all the three designs.

Considering that the fluid bulk mean temperature is the state variable of the reduced heat transfer governing equation of the design layer, the mid-plane temperature field is recovered for comparison purpose using the interface and bulk mean values and temperature profile. Similar temperature distributions on the mid-plane of the design layer are observed from the re-evaluations of the two-layer model and three-dimensional simulations. Fins have much higher temperature than surrounding fluid due to the conductive heat transfer from substrate and effective convection happens between the flow and fins which further promotes the heat dissipation from the substrate. Although the fluid bulk mean temperature is non-negative over the design layer computed by the two-layer model, negative values are observed in the recovered mid-plane temperature fields close to the inlet. This is presumably because the assumption that the flow is hydrodynamically and thermally developed is not fully satisfied in the region close to the inlet, especially under large pressure drops. The real temperature profile at these regions is less smooth than that assumed by Eq. (3). Comparison of the temperature plots of the substrate shows that the two-layer model can also predict the substrate temperature field well. The region near the inlet has lower temperature than other regions. The highest temperature occurs on the opposite side, expect for the temperature field of the design under $200 \mathrm{~Pa}$ pressure drop predicted by the two-layer model. The local regions below the fins have lower temperature than their neighborhood benefiting from the enhancement of heat transfer by the fins. In the designs optimized for pressure drops of $50 \mathrm{~Pa}$ and $200 \mathrm{~Pa}$, higher substrate temperature near the inlet is predicted by the two-layer model than for the threedimensional simulations. Again because the flow is assumed fully developed, the boundary layer 
upon this local region considered in the evaluations by the two-layer model is thicker than that in the three-dimensional simulations. This results in higher interface temperature and higher substrate temperature than predicted.

To quantitatively assess the accuracy of the two-layer heat sink model, the Renolds numbers, objective function values, maximum temperatures over the substrate mid-plane of the designs are compared and shown in Table 2. In the computation of Renolds numbers, the average inlet velocity of the three-dimensional velocity field is used as the characteristic velocity and the width of the whole inlet as the characteristic length. The highest Renolds number is reached at pressure drop of $200 \mathrm{~Pa}$ and is around 630 evaluated by the two-layer model and the three-dimensional simulations. As discussed in [33], the Renolds numbers are well below the critical value for laminar turbulent transition for the flow under consideration, and thereby the assumption of laminar flow is justified. The Renolds numbers evaluated by the two-layer model and three-dimensional simulations are in quite good agreement. Larger deviations between the two modeling strategies are observed in terms of the objective function. For all the designs, the two-layer model consistently predicts higher objective function values and the relative error is below $2.11 \%$ and $7.01 \%$ for pressure drops of $10 \mathrm{~Pa}$ and $50 \mathrm{~Pa}$ respectively, whereas it reaches around $13.27 \% \sim 15.23 \%$ for the pressure drop of $200 \mathrm{~Pa}$. This is mainly due to the fully developed flow assumption being not well satisfied in the local design layer region close to the inlet especially for large pressure drops. It is found that the maximum temperatures estimated by the two approaches have higher deviations than for the objectives. However, the two-layer model is developed primarily to accelerate heat transfer analysis in topology optimization, rather than to provide exact physical fields. Summarizing the comparison of the physical fields and quantitative assessment, the effectiveness of the two-layer model is justified.

\subsection{Influence of parameters}

Taking the design optimized for 50 Pa pressure drop and NSP setting as a benchmark, topology optimizations using different physical and optimization parameter values are carried out to study their effect on optimized layouts. By scaling the values of fluid thermal conductivity, fluid heat capacity, pressure drop and solid inverse permeability, optimized designs are obtained and shown in Figure 8 which have noticeable differences in the layout complexity.

To systematically investigate the influence of parameters, the Peclet numbers indicating the 
Table 2: Comparison between the two-layer model and three-dimensional simulations

(a) Designs of using the Navier-Stokes model and designable inlet and outlet setting

\begin{tabular}{cccccccccc}
\hline \multirow{2}{*}{$\Delta \mathrm{P} / \mathrm{Pa}$} & \multicolumn{3}{c}{ Renolds numbers } & \multicolumn{3}{c}{ Objective values $/{ }^{\circ} \mathrm{C}$} & \multicolumn{3}{c}{ Maximum $\mathrm{T} /{ }^{\circ} \mathrm{C}$} \\
\cline { 2 - 9 } & 2-layer & $3 \mathrm{D}$ & Error & 2-layer & 3D & Error & 2-layer & $3 \mathrm{D}$ & Error \\
\hline 10 & 47.3 & 47.0 & $0.64 \%$ & 62.0 & 61.5 & $0.85 \%$ & 73.5 & 69.8 & $5.34 \%$ \\
50 & 188.6 & 184.7 & $2.11 \%$ & 19.2 & 18.3 & $5.35 \%$ & 22.5 & 20.8 & $8.10 \%$ \\
200 & 573.3 & 524.0 & $9.41 \%$ & 8.9 & 7.9 & $13.27 \%$ & 10.8 & 9.1 & $18.72 \%$ \\
\hline
\end{tabular}

(b) Designs of using the Navier-Stokes model and passive inlet and outlet setting

\begin{tabular}{cccccccccc}
\hline \multirow{2}{*}{$\Delta \mathrm{P} / \mathrm{Pa}$} & \multicolumn{3}{c}{ Renolds numbers } & \multicolumn{3}{c}{ Objective values $/{ }^{\circ} \mathrm{C}$} & \multicolumn{3}{c}{ Maximum $\mathrm{T} /{ }^{\circ} \mathrm{C}$} \\
\cline { 2 - 9 } & 2-layer & $3 \mathrm{D}$ & Error & 2-layer & 3D & Error & 2-layer & $3 \mathrm{D}$ & Error \\
\hline 10 & 48.5 & 48.7 & $0.41 \%$ & 64.1 & 62.8 & $2.11 \%$ & 75.5 & 71.2 & $6.15 \%$ \\
50 & 186.5 & 187.5 & $0.53 \%$ & 19.3 & 18.5 & $4.46 \%$ & 22.7 & 21.0 & $7.84 \%$ \\
200 & 532.6 & 515.9 & $3.24 \%$ & 9.0 & 7.8 & $15.23 \%$ & 10.7 & 9.0 & $19.79 \%$ \\
\hline
\end{tabular}

(c) Designs of using the Stokes model and designable inlet and outlet setting

\begin{tabular}{cccccccccc}
\hline \multirow{2}{*}{$\Delta \mathrm{P} / \mathrm{Pa}$} & \multicolumn{3}{c}{ Renolds numbers } & \multicolumn{3}{c}{ Objective values $/{ }^{\circ} \mathrm{C}$} & \multicolumn{3}{c}{ Maximum $\mathrm{T} /{ }^{\circ} \mathrm{C}$} \\
\cline { 2 - 10 } & 2-layer & 3D & Error & 2-layer & 3D & Error & 2-layer & $3 \mathrm{D}$ & Error \\
\hline 10 & 48.74 & 48.67 & $0.14 \%$ & 63.9 & 63.1 & $1.28 \%$ & 75.4 & 71.0 & $6.11 \%$ \\
50 & 198.7 & 198.9 & $0.10 \%$ & 18.4 & 17.2 & $7.01 \%$ & 21.6 & 19.8 & $8.79 \%$ \\
200 & 616.3 & 614.9 & $0.23 \%$ & 8.3 & 7.3 & $13.84 \%$ & 9.4 & 8.4 & $11.62 \%$ \\
\hline
\end{tabular}

(d) Designs of using the Stokes model and passive inlet and outlet setting

\begin{tabular}{cccccccccc}
\hline \multirow{2}{*}{$\Delta \mathrm{P} / \mathrm{Pa}$} & \multicolumn{3}{c}{ Renolds numbers } & \multicolumn{3}{c}{ Objective values $/{ }^{\circ} \mathrm{C}$} & \multicolumn{3}{c}{ Maximum $\mathrm{T} /{ }^{\circ} \mathrm{C}$} \\
\cline { 2 - 9 } & 2-layer & $3 \mathrm{D}$ & Error & 2-layer & 3D & Error & 2-layer & $3 \mathrm{D}$ & Error \\
\hline 10 & 48.7 & 48.6 & $0.21 \%$ & 63.8 & 63.0 & $1.25 \%$ & 75.2 & 70.9 & $6.12 \%$ \\
50 & 195.4 & 195.3 & $0.05 \%$ & 18.4 & 17.3 & $6.50 \%$ & 21.6 & 19.9 & $8.35 \%$ \\
200 & 633.7 & 627.3 & $1.02 \%$ & 7.7 & 6.7 & $14.77 \%$ & 9.3 & 8.3 & $12.15 \%$ \\
\hline
\end{tabular}




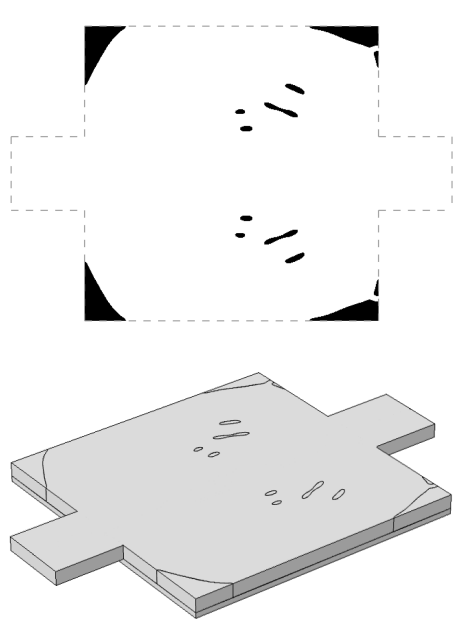

(a)

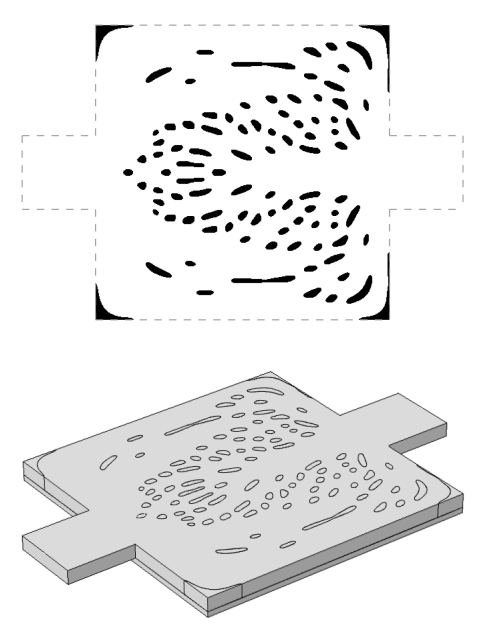

(b)

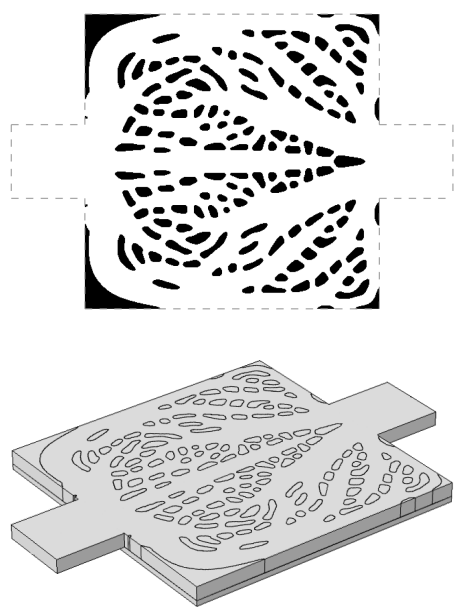

(c)

Figure 6: Optimized designs and the corresponding three-dimensional heat sinks used for model validation. Specifically, designs of NSP setting and pressure drops of (a) $10 \mathrm{~Pa}$, (b) $50 \mathrm{~Pa}$ and (c) $200 \mathrm{~Pa}$ are used.

relation between advective and diffusive heat transfer are compared among the designs. The Peclet numbers are evaluated by $\mathrm{Pe}=\frac{L_{c} U_{c}}{k_{f} /\left(\rho_{0} C\right)}$, where the width of the inlet is used as the characteristic length $L_{c}$ and the characteristic velocity is computed by $U_{c}=\sqrt{\Delta \mathrm{P} / \rho_{0}}$ [17. since the pressure drop is prescribed in the optimization. Compared to the benchmark, designs shown in Figure 8 (a)-(c) have more complicated channel networks and higher Peclet numbers. Designs (d) and (e) have similar layouts to the benchmark and the same Peclet numbers. Design (f) has the simplest channel layout consisting of six small pin fins and two plate fins only and lowest Peclet number. Hence optimized designs are more likely to have complex layouts when large Peclet numbers are assigned. To acquire a general insight into the influence of the parameters, more studies are needed which is beyond the scope of this work. We notice, however, that a similar yet more thorough study on the influence of parameters has been done for the topology optimization of two-dimensional catalytic microfluidic reactors in [46].

\subsection{Designs at low pressure drops}

By allowing the whole thermal-fluid layer to be designable, the optimized design for a pressure drop of 3.5 Pa and using the Navier-Stokes flow model is obtained as in Figure 9 (a). Without thinking too much about the physical realization of the boundary conditions, it is observed that heat is transferred away partly through convection and partly through conduction to the inlet. As 
(a)

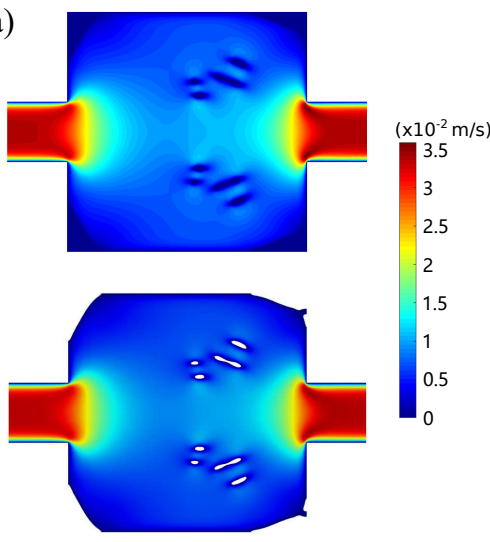

(b)

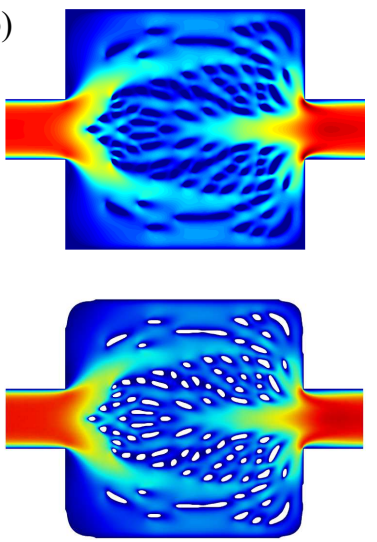

(c)
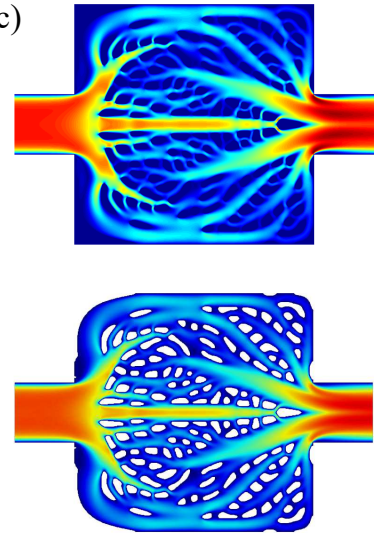
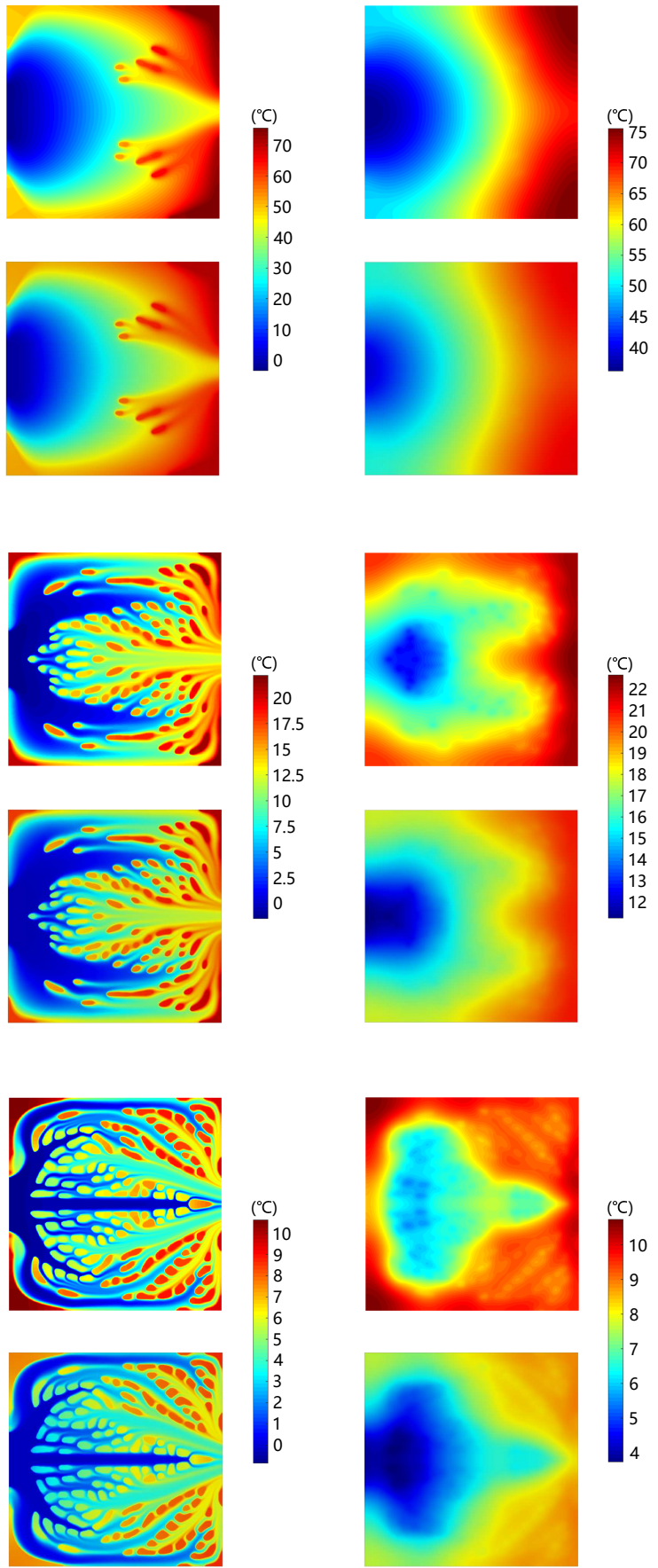

Figure 7: Physical fields of optimized designs using the NSP setting and pressure drops of $10 \mathrm{~Pa}(\mathrm{a}), 50 \mathrm{~Pa}$ (b) and $200 \mathrm{~Pa}$ (c). The upper sub-figures in (a)-(c) are the velocity magnitude field (left), temperature field (middle) on the mid-plane of design layer and temperature field on the mid-plane of substrate (right) computed by the developed two-layer heat sink model. The lower sub-figures in (a)-(c) are the physics fields of the three-dimensional simulations in Comsol Multiphysics. 


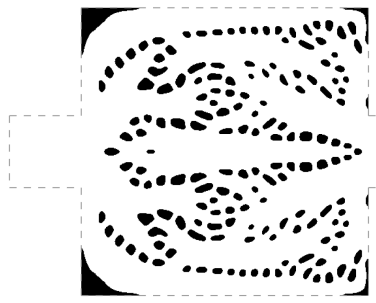

(a) $\mathrm{Pe}=3.51 \times 10^{4}$

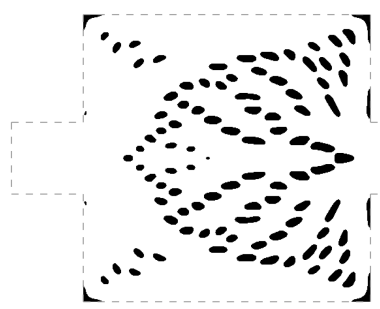

(d) $\mathrm{Pe}=3.90 \times 10^{3}$

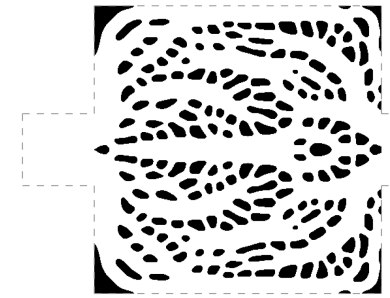

(b) $\mathrm{Pe}=3.51 \times 10^{4}$

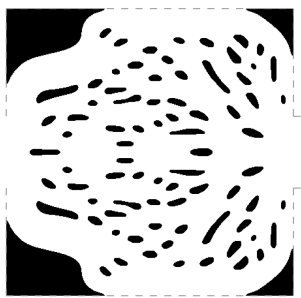

(e) $\mathrm{Pe}=3.90 \times 10^{3}$

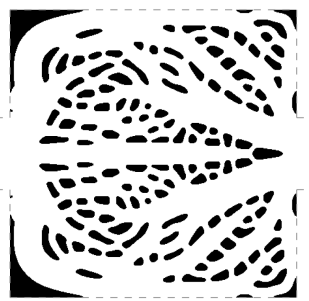

(c) $\mathrm{Pe}=7.81 \times 10^{3}$

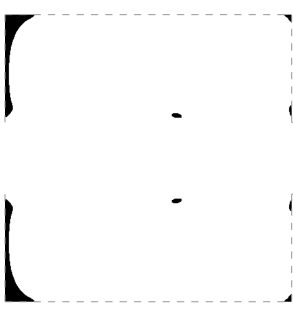

(f) $\mathrm{Pe}=4.34 \times 10^{2}$

Figure 8: Designs optimized for $50 \mathrm{~Pa}$ pressure drop and NSP setting with different physical and optimization parameter values. Compared to the counterpart design in Figure 3 where the Peclet number $\mathrm{Pe}=3.90 \times 10^{3}$, the following parameters are set as (a) $k_{f}=k_{f 0} / 9$, (b) $C=9 C_{0}$, (c) $\Delta \mathrm{P}=200 \mathrm{~Pa}$, (d) $\alpha_{s}=500 \alpha_{f 0}$, (e) $C=4 C_{0}$, $k_{f}=4 k_{f 0}$, (f) $C=C_{0} / 9$, where $\square_{0}$ denotes the parameters used for the design in Figure 3

the imposed pressure drop decreases, it is expected that more heat is dissipated in the form of conduction in the designs until it becomes actually a heat conduction problem due to the negligible convection effect. On the other hand, the convection effect can also be reduced by changing the material properties of solid and fluid while keeping the pressure drop at $3.5 \mathrm{~Pa}$. An example is reducing the heat capacity of fluid to $C=C_{0} / 4$ and increasing the thermal conductivity of solid to $k_{s}=4 k_{s 0}$ where $\square_{0}$ denotes the original material properties given in Table 1. Further restricting the volume fraction of fluid no smaller than that of the design in Figure 9 (a), a tree structure is obtained as shown in Figure 9 (b) which is a typical topology of the local minima commonly seen in the topology optimization of heat conduction structures. Considering that rank-1 laminates [47] are optimal for heat conduction problems as discussed by Yan et al. [13, the topology optimization of a two-layer heat conduction structure is carried out using rank-1 laminates by neglecting the convection term in the governing equations (7). Large regions of intermediate densities exist in the obtained rank-1 design. Applying the projection approach proposed in [48] to the rank-1 design, a discrete needle structure which is a macroscopic approximation of the periodic microscopic rank-1 laminates is obtained as shown in Figure 9 (c). Re-analyzing the needle structure using the two- 
layer heat sink model shows a more uniform temperature distribution and lower values than for the tree structure. As the flow in the tree and needle structures as well as the convection effect are negligible, the heat transfer has become a heat conduction process. The comparison of designs in Figure 9 (b) and (c) is then in line with the study in [13] on the non-optimality of tree structures for heat conduction problems.

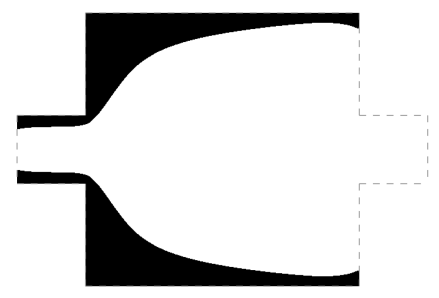

(a)

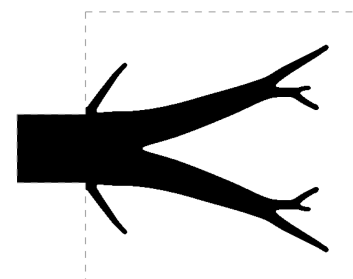

(b)

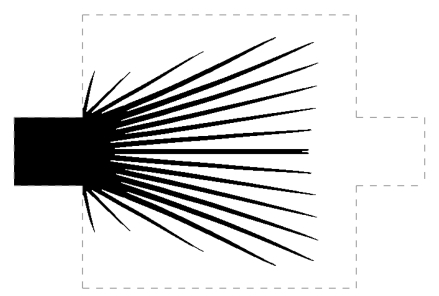

(c)

Figure 9: Designs for a pressure drop of 3.5 Pa and using Navier-Stokes flow model, when the whole design layer is designable. (a) Optimized design using the material properties given in Table 1 (b) Tree structure obtained by using fluid heat capacity $C=C_{0} / 4$ and solid thermal conductivity $k_{s}=4 k_{s 0}$ where $\square_{0}$ denotes the values given in Table 1 (c) Discrete needle structure projected from the rank-1 design of the two-layer heat conduction problem.

\subsection{Comparison with one-layer model}

In the literature on topology optimization of heat sinks, a one-layer model is widely used in which the substrate is omitted and the heat flux is applied directly to the thermal-fluid layer. The fluid and solid regions are also distinguished from each other through the force term in the fluid dynamics equations, but a zero force is set in the fluid region. The one-layer model can be expressed as

Fluid dynamics equations:

$$
\begin{aligned}
& \nabla \cdot \mathbf{u}=0 \\
& \rho_{0} \mathbf{u} \cdot \nabla \mathbf{u}=-\nabla P+\mu \nabla \cdot\left(\nabla \mathbf{u}+(\nabla \mathbf{u})^{\mathrm{T}}\right)-\alpha(\gamma) \mathbf{u}
\end{aligned}
$$

Heat transfer equation:

$$
\gamma \rho_{0} C \mathbf{u} \cdot \nabla T=\nabla \cdot(k(\gamma) \nabla T)+\frac{q^{\prime \prime}(\gamma)}{2 H_{t}}
$$

where, the inverse permeability $\alpha$ is zero in fluid region and can be computed by $\alpha=\mu /\left(\mathrm{Da} \cdot L_{c}^{2}\right)$ 33. in solid region with the Darcy number set as $\mathrm{Da}=10^{-4}, 10^{-5}, 10^{-6}$ respectively [49] and the inlet width as the characteristic length $L_{c}$, the effective thermal conductivity takes that of fluid and solid in corresponding regions, the imposed heat flux $q^{\prime \prime}(\gamma)$ is design-independent or 
dependent according to the assumptions on the redistribution of heat flux by the omitted substrate. If uniform heat transfer from substrate to fins and fluid is assumed, $q^{\prime \prime}(\gamma)=q_{0}^{\prime \prime}$ is design-independent. In comparison, if the imposed heat flux is assumed to transfer to fins only, $q^{\prime \prime}(\gamma)=q_{0}^{\prime \prime} A_{\Omega}(1-\gamma) / \iint_{\Omega}(1-\gamma) d \Omega$ where $A_{\Omega}$ is the area of the square flow region in design layer and $\iint_{\Omega}(1-\gamma) d \Omega$ is the area occupied by fins in the square region.

To compare with the developed two-layer model, the design optimized for the pressure drop of $50 \mathrm{~Pa}$ and NSP setting is re-evaluated by the one-layer model with design-independent and dependent heat flux distributions, respectively. It is noticed that whether or not the design variable $\gamma$ is included in the convection term in the heat transfer equation should not influence the reevaluation results. Re-evaluations using the same setting of inverse permeability as in the two-layer model, i.e. $\alpha=5 \mu /\left(2 H_{t}^{2}\right)$ in fluid region and $\alpha=5 \mu /\left[2\left(H_{t} / 100\right)^{2}\right]$ in solid region, are also carried out. The effect of the thermal coupling between thermal-fluid layer and substrate can then be studied. Keeping in mind that the problem is linear with respect to the imposed heat flux, $q_{0}^{\prime \prime}=2.5 \times 10^{4} \mathrm{~W} / \mathrm{m}^{2}$ is used in the re-evaluations in order to obtain results with physical meaning. The obtained velocity magnitude fields and temperature fields are shown in Figure 10 The velocity and temperature fields of the one-layer model, two-layer model and three-dimensional simulations are compared. Obvious differences in both velocity distribution and temperature distribution are observed. As shown in Figure 10 (a)-(c), fluid flow at higher velocity is obtained as a result of neglecting the out-of-plane force when the inverse permeability in the fluid region is zero. The fluid flowing through the bypasses beside the inlet is reduced due to the inertia effect, which weakens the convective heat transfer in these local regions. Higher temperatures are then obtained compared to the corresponding fields in Figure 10 (d) which are obtained by using the same inverse permeability setting as in the two-layer model. In the temperature fields in Figure 10 , the fins at the corners have much higher temperature than those in the middle region, resulting from the lack of convective cooling while imposed with the same heat flux. Therefore, the one-layer model fails to predict the temperature distribution in heat sinks properly due to the simple treatment of the thermal coupling between design layer and substrate, while the developed two-layer model is more adequate in predicting the real heat transfer process as discussed in Section 5.2. 

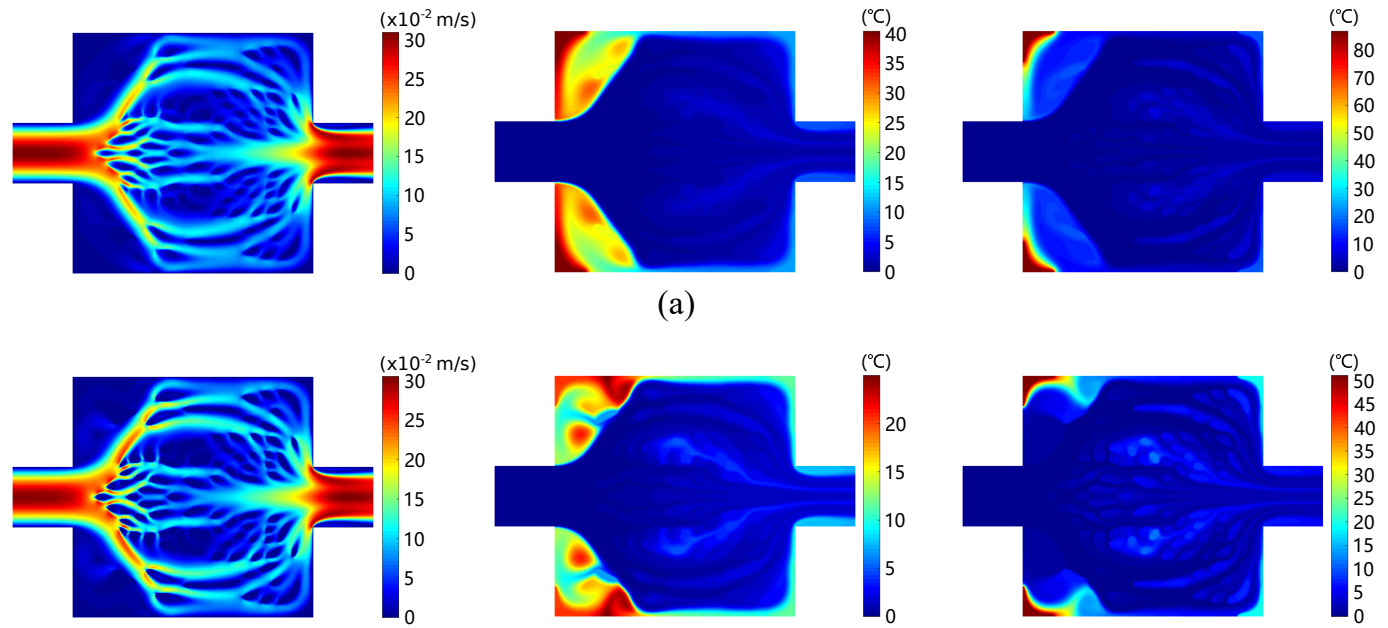

(b)
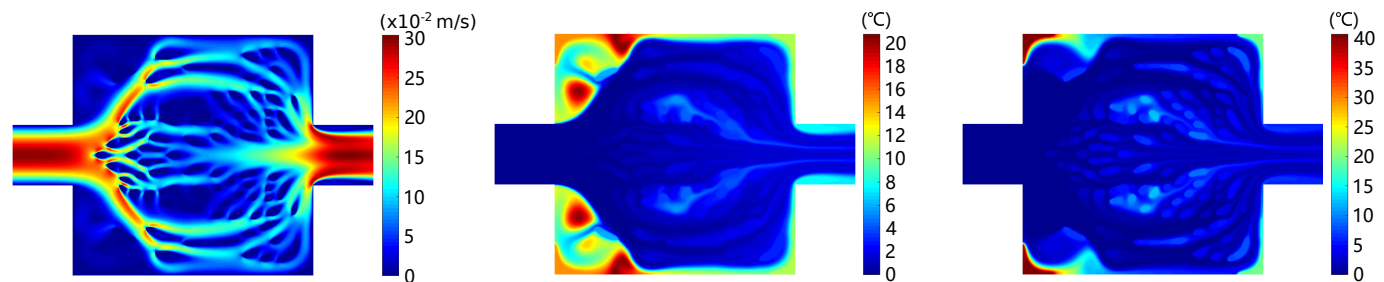

(c)
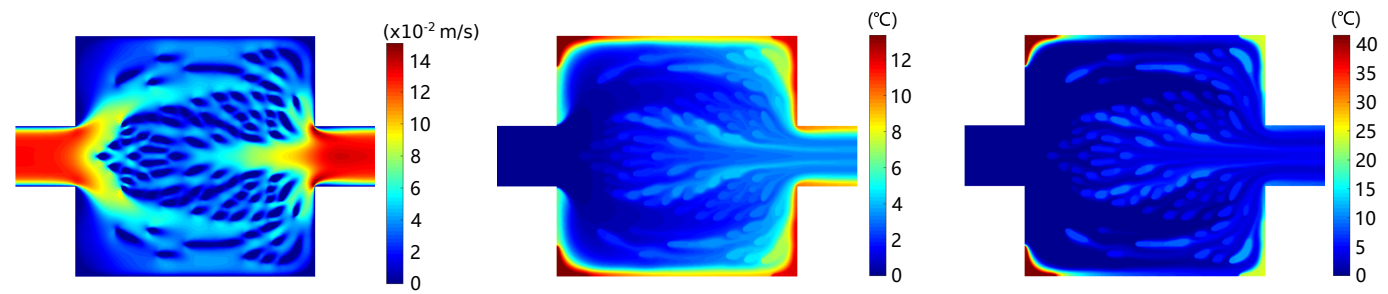

(d)

Figure 10: Physical fields from the re-evaluation by the one-layer model of the design optimized for 50 Pa pressure drop and NSP setting. The Darcy number is set as $\mathrm{Da}=10^{-4}$ in (a), $10^{-5}$ in (b) and $10^{-6}$ in (c), while the inverse permeability setting in $(\mathrm{d})$ is the same as that in the two-layer model. The sub-figures from left to right are velocity magnitude field, temperature field under design-independent heat flux and temperature field under dependent heat flux, respectively. 


\section{Conclusions}

In this paper, we investigate the topology optimization of microchannel heat sinks at low aspect ratios. To perform convincing topology optimizations of heat sinks at close to two-dimensional computational cost, a two-layer heat sink model is developed with which the full three-dimensional conjugate heat transfer analysis is avoided. As the first step of deriving the two-layer model, the characteristics of the velocity and temperature distributions are studied for the heat sinks under consideration. Reduced two-dimensional fluid dynamics equations proposed in the literature is adopted which is developed assuming a parabolic velocity profile. A fourth-order polynomial temperature profile of the thermal-fluid layer and a linear temperature profile of the substrate are derived by making suitable assumptions. By applying the velocity and temperature profiles, the dependency of the heat transfer equations on the height direction is eliminated and some rescaling constants are introduced. The thermal coupling between the thermal-fluid layer and the substrate is realized through an out-of-plane heat flux term where the heat transfer coefficient is determined based on the conservation of heat flow between layers. The reduced two-dimensional heat transfer equations are therefore coupled to one system.

Using the developed two-layer model, topology optimizations of a square heat sink are performed for different prescribed pressure drop values, design domain settings and flow models with and without the inertia term. As expected, the layout of designs becomes more complex with increasing pressure drop. The inertia effect can result in quite different designs when the pressure drop is large, such as $200 \mathrm{~Pa}$ as used in this work and a resulting Renolds number of more than 500. Cross-check of the optimized designs is done to validate the proposed two-layer model and implementation strategies. The accuracy of the two-layer model is further examined by comparing to three-dimensional conjugate heat transfer analysis of the optimized designs in COMSOL Multiphysics and a good agreement between the results is observed. The influence of physical and optimization parameters is also investigated and related to the Peclet number. Topology optimizations of heat sinks at a very low pressure drop are carried out for academic interest. As the convective heat transfer becomes negligible, a tree structure and a lamellar needle structure are obtained which constitute the typical local optimum and (near-)global optimum for heat conduction problems respectively. One of the designs optimized for the $50 \mathrm{~Pa}$ pressure drop is re-evaluated by using the one-layer heat sink model which is commonly seen in literature. Comparison to the corresponding three-dimensional simulation shows that the one-layer model fails to predict the velocity and temperature fields properly 
and hence unsuitable for the considered problems.

\section{Acknowledgements}

FW and OS acknowledge the support of the Villum Fonden through the Villum investigator project InnoTop. Part of this work was performed while SY was a visiting student at the Department of Mechanical Engineering, Technical University of Denmark, supported by the China Scholarship Council (CSC). SY acknowledges support from the Fok Ying Tong Education Foundation for Young Teachers in the Higher Education Institutions of China (161047).

\section{Appendix A. Derivations of the two-layer heat sink model}

Detailed derivations of the two-layer model used for the topology optimization of heat sinks are given in this appendix. The heat sinks under consideration are as the one shown in Figure 1 which have quite large in-plane $(x-y)$ dimensions compared with their height. The approach used here is inspired by the reduction of the fluid dynamics equations of three-dimensional plane flows in [15, 16. The temperature profiles of the thermal-fluid design layer and substrate are derived at first by making some suitable assumptions. Based on these profiles, the weak forms of the heat transfer governing equations are integrated and then localized to give the reduced governing equations.

\section{A.1. Temperature profile in the thermal-fluid design layer}

Keeping the assumptions on the flow and heat transfer process made in Section 2 in mind, we consider the special case where the design layer consists of flow only and simplify the flow as a Poiseuille flow between two parallel plates. Denoting the flow direction as $x$-direction and the height of heat sink as $z$-direction, then according to Eq. (1) the three-dimensional velocity field of the flow in design layer is

$$
\bar{u}_{1}=f_{1}(z) u_{1}=\left[1-\left(\frac{z}{H_{t}}\right)^{2}\right] u_{1}, \quad \bar{u}_{2}=0, \quad \bar{u}_{3}=0
$$

where, $\bar{u}_{i}(i=1,2,3)$ are the components of the three-dimensional velocity vector and $\mathbf{u}=\left[u_{1}, 0\right]^{\mathrm{T}}$ represents the two-dimensional velocity field in the mid-plane of design layer. 
Supposing that the axial conduction is negligible [4, then the heat transfer governing equation of the thermal-fluid flow can be simplified as

$$
\rho_{0} C\left(\bar{u}_{1} \frac{\partial \bar{T}_{t}}{\partial x}\right)=\frac{\partial}{\partial z}\left(k_{f} \frac{\partial \bar{T}_{t}}{\partial z}\right)
$$

Similar to the velocity distribution, the profile of the non-dimensional temperature variable $\theta_{t}$ defined in Section 2.1 is also invariant in the flow direction for fully developed flow. In the following, the profile of $\theta_{t}$, i.e., $f_{2}(z)$, is derived which provides a basis for reducing the governing equation and also for recovering three-dimensional temperature field from the two-dimensional one. The partial derivatives of $\theta_{t}$ are computed as

$$
\frac{\partial \theta_{t}}{\partial x}=\frac{\partial}{\partial x}\left(\frac{T_{i}-\bar{T}_{t}}{T_{i}-T_{t}}\right)=0, \quad \frac{\partial \theta_{t}}{\partial z}=-\frac{\partial \bar{T}_{t} / \partial z}{T_{i}-T_{t}}=\frac{\partial f_{2}(z)}{\partial z}
$$

Considering the thermal energy balance on the bottom of design layer, we have

$$
q_{i}^{\prime \prime}=h_{t}\left(T_{i}-T_{t}\right)=-k_{f}\left(\frac{\partial \bar{T}_{t}}{\partial z}\right)_{z=-H_{t}}
$$

where, $q_{i}^{\prime \prime}$ is the out-of-plane heat flux imposed by the substrate through the interface representing the thermal coupling between design layer and substrate and $h_{t}$ is the heat transfer coefficient. By combining the second relation in Eq. 20, and Eq.21, we obtain that $h_{t} / k_{f}$ is constant. As the thermal conductivity of fluid $k_{f}$ is constant, $h_{t}$ is also constant over the interface. Supposing that the out-of-plane heat flux applied at the interface can be considered as constant, i.e., $q_{i}^{\prime \prime}=$ $h_{t}\left(T_{i}-T_{t}\right)=$ const, then $\left(T_{i}-T_{t}\right)$ is constant, from which $\mathrm{d} T_{i} / \mathrm{d} x=\mathrm{d} T_{t} / \mathrm{d} x$. Substituting these relations into the first equation of Eq. 200 gives

$$
\frac{\partial \bar{T}_{t}}{\partial x}=\frac{\mathrm{d} T_{i}}{\mathrm{~d} x}=\frac{\mathrm{d} T_{t}}{\mathrm{~d} x}
$$

Substituting the definition of $\theta_{t}$ in Eq. (3) and Eq. 22, into Eq. 197, we obtain the following relation after some algebra,

$$
\frac{\mathrm{d}^{2} f_{2}(z)}{\mathrm{d} z^{2}}=\frac{\rho_{0} C \mathrm{~d} T_{t} / \mathrm{d} x}{k_{f}\left(T_{t}-T_{i}\right)} \cdot \bar{u}_{1}
$$

Integrating Eq. (23) twice with respect to $z$ and applying the velocity profile in Eq. (18), we obtain 
that

$$
f_{2}(z)=\frac{\lambda}{13}\left[\left(\frac{z}{H_{t}}\right)^{4}-6\left(\frac{z}{H_{t}}\right)^{2}+8\left(\frac{z}{H_{t}}\right)+13\right]
$$

based on the boundary conditions

$$
\bar{T}_{t}=T_{i} \text { at } z=-H_{t}, \quad \frac{\partial \bar{T}_{t}}{\partial z}=0 \text { at } z=H_{t}
$$

i.e.,

$$
f_{2}(z)=0 \text { at } z=-H_{t}, \quad \frac{\mathrm{d} f_{2}}{\mathrm{~d} z}=0 \text { at } z=H_{t}
$$

To decide the value of $\lambda$ in the above profile of $\theta_{t}$, we rewrite the expression of the bulk mean temperature here.

$$
T_{t}=\frac{1}{2 H_{t} u_{m}} \int_{-H_{t}}^{H_{t}}\|\overline{\mathbf{u}}\| \bar{T}_{t} \mathrm{~d} z, \text { where } u_{m}=\frac{1}{2 H_{t}} \int_{-H_{t}}^{H_{t}}\|\overline{\mathbf{u}}\| \mathrm{d} z
$$

Replacing $\bar{T}_{t}$ in the above expression by $T_{i}, T_{t}$ and $f_{2}(z)$ according to Eq. (3) and using the velocity profile in Eq. 18 gives the relation of velocity profile and temperature profile

$$
\int_{-H_{t}}^{H_{t}} f_{1}(z) f_{2}(z) \mathrm{d} z=\frac{4}{3} H_{t}
$$

The profile of the non-dimensional temperature variable $\theta_{t}$ is then obtained,

$$
f_{2}(z)=\frac{35}{416}\left[\left(\frac{z}{H_{t}}\right)^{4}-6\left(\frac{z}{H_{t}}\right)^{2}+8\left(\frac{z}{H_{t}}\right)+13\right], \quad z \in\left[-H_{t}, H_{t}\right]
$$

The three-dimensional temperature field can be recovered through equation

$$
\bar{T}_{t}=T_{i}-f_{2}(z)\left(T_{i}-T_{t}\right)
$$

\section{A.2. Reduction of the heat transfer equation for thermal-fluid design layer}

Denoting the design layer as $\bar{\Omega}_{t}=\left\{\mathbf{x}=(x, y, z) \in \mathbf{R}^{3} \mid(x, y) \in \Omega_{t},-H_{\mathrm{t}} \leq z \leq H_{\mathrm{t}}\right\}$ where $\Omega_{t} \subset$ $\mathbf{R}^{2}$ represents the in-plane $(x-y)$ region occupied by the design layer, the complete three-dimensional 
heat transfer governing equation of the design layer is

$$
\rho_{0} C\left(\overline{\mathbf{u}} \cdot \nabla \bar{T}_{t}\right)-\nabla \cdot\left(k_{f} \nabla \bar{T}_{t}\right)=0
$$

with the boundary conditions

$$
\bar{T}_{t}=T_{0} \text { on } \bar{\Gamma}_{i n}, \quad \bar{T}_{t}=T_{i} \text { on } \bar{\Gamma}_{i t}, \quad-k_{f} \frac{\partial \bar{T}_{t}}{\partial n}=0 \text { on } \bar{\Gamma}_{t}
$$

where $T_{0}$ is the prescribed fluid temperature at the inlet boundary $\bar{\Gamma}_{i n}, T_{i}$ is the temperature at the interface between design layer and substrate $\bar{\Gamma}_{i t}, \bar{\Gamma}_{t}$ represents the rest boundaries of design layer.

The weak form the governing equation 28 is: find $\bar{T}_{t} \in \bar{S}$ such that

$$
\int_{\bar{\Omega}_{t}} \rho_{0} C \bar{\varphi}\left(\overline{\mathbf{u}} \cdot \nabla \bar{T}_{t}\right) d V+\int_{\bar{\Omega}_{t}} k_{f} \nabla \bar{\varphi} \cdot \nabla \bar{T}_{t} d V=0 \quad \forall \bar{\varphi} \in \bar{\Pi}
$$

where, $\bar{S}$ and $\bar{\Pi}$ are the admissible spaces for the temperature field $\bar{T}_{t}$ and weight function $\bar{\varphi}$, respectively. Considering that the temperature field has a profile of Eq. 26], the weight function is assumed to have the form $\bar{\varphi}(x, y, z)=f_{2}(z) \varphi(x, y)$ where $\varphi$ is the corresponding two-dimensional weight function. As a result, the profile functions $f_{1}(z)$ and $f_{2}(z)$ are the only terms depending on $z$ in the above weak formulation. Integrating the weak formulation with respect to $z$, some rescaling constants are introduced and using the relation $\nabla T_{i}=\nabla T_{t}$, we obtain

$$
\int_{\Omega_{t}} \varphi\left[\frac{4 H_{t}}{3} \rho_{0} C\left(\mathbf{u} \cdot \nabla T_{t}\right)-\frac{49 H_{t}}{26} \nabla \cdot\left(k_{f} \nabla T_{t}\right)-\frac{35 k_{f}}{26 H_{t}}\left(T_{i}-T_{t}\right)\right] \mathrm{d} \Omega=0
$$

Considering the arbitrariness of function $\varphi$, the above weak formulation is localized and the reduced heat transfer governing equation defined on $\Omega_{t}$ is obtained,

$$
\frac{4 H_{t}}{3} \rho_{0} C\left(\mathbf{u} \cdot \nabla T_{t}\right)-\frac{49 H_{t}}{26} \nabla \cdot\left(k_{f} \nabla T_{t}\right)-\frac{35 k_{f}}{26 H_{t}}\left(T_{i}-T_{t}\right)=0
$$

with boundary conditions

$$
T_{t}=T_{0} \text { on } \Gamma_{i n}, \quad-k_{f} \frac{\partial T_{t}}{\partial n}=0 \text { on } \Gamma_{t}
$$

where, $\Gamma_{i n}$ is the corresponding two-dimensional inlet boundary and $\Gamma_{t}$ is the rest boundaries of $\Omega_{t}$. Replacing $\bar{T}_{t}$ in the energy balance equation 21 by the two-dimensional temperatures and the 
profile $f_{2}(z)$, we obtain

$$
h_{t}=\frac{35 k_{f}}{26 H_{t}}
$$

Therefore, the third term on the left of the reduced governing equation (31) is actually the outof-plane heat flux $q_{i}^{\prime \prime}$. The Nussel number at the interface between the thermal-fluid layer and substrate is then

$$
\mathrm{Nu}=\frac{h_{t} D}{k_{f}}=\frac{35 D}{26 H_{t}}
$$

where, $D$ is the characteristic length which is set as the hydraulic diameter of parallel plate channels. Hence, $D=4 H_{t}$ and the obtained Nusselt number is $\mathrm{Nu}=5.3846$ which is consistent with the classical result in [50].

In topology optimization process, the material distribution in design layer keeps changing and intermediate design variables exist. Thus the effective thermal conductivity $k_{t}=k_{t}(\gamma)$ defined in Section 4.1 is used instead of the fluid conductivity $k_{f}$ and the above reduced convection-diffusion equation is reformulated as

$$
\frac{2}{3} \rho_{0} C\left(\mathbf{u} \cdot \nabla T_{t}\right)-\frac{49}{52} \nabla \cdot\left(k_{t} \nabla T_{t}\right)-\frac{1}{2 H_{t}} \cdot h_{t}\left(T_{i}-T_{t}\right)=0
$$

\section{A.3. Reduction of the heat transfer equation for substrate}

Assuming the axial heat conduction is negligible in the substrate compared to $z$-directional conduction, the heat transfer governing equation is then simplified as

$$
-\frac{\partial}{\partial z}\left(k_{b} \frac{\partial \bar{T}_{b}}{\partial z}\right)=0
$$

By integrating this simplified governing equation twice with respect to $z$ and applying the boundary conditions

$$
\bar{T}_{b}=T_{i} \text { at } z=H_{b}, \quad-k_{b} \frac{\partial \bar{T}_{b}}{\partial z}=q_{0}^{\prime \prime} \text { at } z=-H_{b}
$$

the temperature profile of the substrate is obtained which is a linear function of $z$ as follows.

$$
\bar{T}_{b}=T_{i}+\frac{q_{0}^{\prime \prime} H_{b}}{k_{b}}\left(1-\frac{z}{H_{b}}\right)
$$


Then the profile of the non-dimensional temperature variable $\theta_{b}$ for substrate defined in Section 2.2 is obtained as a function of $z$ only.

Based on the profile of the non-dimensional temperature variable $\theta_{b}$, the three-dimensional governing equation of substrate can be reduced by constructing the weak formulation, integrating to eliminate $z$ and then localizing the reduced weak formulation. The obtained reduced governing equation is

$$
-\frac{k_{b}}{2} \nabla^{2} T_{b}+\frac{1}{2 H_{b}} \cdot h_{b}\left(T_{b}-T_{i}\right)-\frac{q_{0}^{\prime \prime}}{2 H_{b}}=0
$$

where, the heat transfer coefficient $h_{b}=k_{b} / H_{b}$ and the out-of-plane heat flux which couples the thermal-fluid layer to substrate can also be denoted as $q_{i}^{\prime \prime}=h_{b}\left(T_{b}-T_{i}\right)$.

To eliminate the unknown interface temperature $T_{i}$, a new heat transfer coefficient is introduced for the thermal circuit in series as explained in Section 2.2 and the two-layer heat transfer governing equations of the heat sink can then be formulated as Eq. (7). From above derivations, it is noted that the two-layer heat sink model is more accurate when the design layer only contains fluid at relatively low velocity and the fourth-order polynomial temperature profile does not apply in the fin regions. Hence, the two-layer model cannot be used to estimate the exact temperature values, but it can resolve the heat transfer at a certain accuracy. Considering that the objective of developing the two-layer model is to do topology optimization of heat sinks at a low computational cost, the model is still good as demonstrated by the comparison with three-dimensional simulations in Comsol Multiphysics in Section 5.2.

\section{References}

[1] D. B. Tuckerman, R. F. W. Pease, High-performance heat sinking for vlsi, IEEE Electron device letters 2 (5) (1981) 126-129.

[2] S. Kim, D. Kim, Forced convection in microstructures for electronic equipment cooling, Journal of Heat Transfer 121 (3) (1999) 639-645.

[3] D.-K. Kim, S. J. Kim, Averaging approach for microchannel heat sinks subject to the uniform wall temperature condition, International Journal of Heat and Mass Transfer 49 (3-4) (2006) 695-706.

[4] D. Liu, S. V. Garimella, Analysis and optimization of the thermal performance of microchannel heat sinks, in: ASME 2003 International Electronic Packaging Technical Conference and Exhibition, American Society of Mechanical Engineers, 2003, pp. 557-565.

[5] G. Fabbri, Optimization of heat transfer through finned dissipators cooled by laminar flow, International journal of heat and fluid flow 19 (6) (1998) 644-654.

[6] J. Tullius, T. K. Tullius, Y. Bayazitoglu, Optimization of short micro pin fins in minichannels, International Journal of Heat and Mass Transfer 55 (15-16) (2012) 3921-3932. 
[7] T. Ambreen, M.-H. Kim, Effect of fin shape on the thermal performance of nanofluid-cooled micro pin-fin heat sinks, International Journal of Heat and Mass Transfer 126 (2018) 245-256.

[8] H. E. Ahmed, B. Salman, A. S. Kherbeet, M. Ahmed, Optimization of thermal design of heat sinks: A review, International Journal of Heat and Mass Transfer 118 (2018) 129-153.

[9] M. P. Bendsøe, N. Kikuchi, Generating optimal topologies in structural design using a homogenization method, Computer methods in applied mechanics and engineering 71 (2) (1988) 197-224.

[10] M. P. Bendsøe, Optimal shape design as a material distribution problem, Structural optimization 1 (4) (1989) 193-202.

[11] G. I. Rozvany, M. Zhou, T. Birker, Generalized shape optimization without homogenization, Structural and Multidisciplinary Optimization 4 (3) (1992) 250-252.

[12] M. Stolpe, K. Svanberg, An alternative interpolation scheme for minimum compliance topology optimization, Structural and Multidisciplinary Optimization 22 (2) (2001) 116-124.

[13] S. Yan, F. Wang, O. Sigmund, On the non-optimality of tree structures for heat conduction, International Journal of Heat and Mass Transfer 122 (2018) 660-680.

[14] M. C. E. Manuel, P. T. Lin, Design explorations of heat conductive pathways, International Journal of Heat and Mass Transfer 104 (2017) 835-851.

[15] T. Borrvall, J. Petersson, Topology optimization of fluids in stokes flow, International journal for numerical methods in fluids 41 (1) (2003) 77-107.

[16] A. Gersborg-Hansen, O. Sigmund, R. B. Haber, Topology optimization of channel flow problems, Structural and Multidisciplinary Optimization 30 (3) (2005) 181-192.

[17] K. Yaji, S. Yamasaki, S. Tsushima, T. Suzuki, K. Fujita, Topology optimization for the design of flow fields in a redox flow battery, Structural and Multidisciplinary Optimization 57 (2) (2018) 535-546.

[18] O. Sigmund, Design of multiphysics actuators using topology optimization-part i: One-material structures, Computer methods in applied mechanics and engineering 190 (49-50) (2001) 6577-6604.

[19] O. Sigmund, Design of multiphysics actuators using topology optimization-part ii: Two-material structures, Computer methods in applied mechanics and engineering 190 (49-50) (2001) 6605-6627.

[20] J. Alexandersen, O. Sigmund, N. Aage, Large scale three-dimensional topology optimisation of heat sinks cooled by natural convection, International Journal of Heat and Mass Transfer 100 (2016) 876891.

[21] J. Alexandersen, O. Sigmund, K. E. Meyer, B. S. Lazarov, Design of passive coolers for light-emitting diode lamps using topology optimisation, International Journal of Heat and Mass Transfer 122 (2018) 138-149.

[22] E. M. Dede, S. N. Joshi, F. Zhou, Topology optimization, additive layer manufacturing, and experimental testing of an air-cooled heat sink, Journal of Mechanical Design 137 (11) (2015) 111403.

[23] A. Iga, S. Nishiwaki, K. Izui, M. Yoshimura, Topology optimization for thermal conductors considering design-dependent effects, including heat conduction and convection, International Journal of Heat and Mass Transfer 52 (11-12) (2009) 2721-2732.

[24] P. Coffin, K. Maute, Level set topology optimization of cooling and heating devices using a simplified convection model, Structural and multidisciplinary optimization 53 (5) (2016) 985-1003. 
[25] J. Alexandersen, N. Aage, C. S. Andreasen, O. Sigmund, Topology optimisation for natural convection problems, International Journal for Numerical Methods in Fluids 76 (10) (2014) 699-721.

[26] E. M. Dede, Optimization and design of a multipass branching microchannel heat sink for electronics cooling, Journal of Electronic Packaging 134 (4) (2012) 041001.

[27] T. Matsumori, T. Kondoh, A. Kawamoto, T. Nomura, Topology optimization for fluid-thermal interaction problems under constant input power, Structural and Multidisciplinary Optimization 47 (4) (2013) 571-581.

[28] A. A. Koga, E. C. C. Lopes, H. F. V. Nova, C. R. De Lima, E. C. N. Silva, Development of heat sink device by using topology optimization, International Journal of Heat and Mass Transfer 64 (2013) $759-772$.

[29] K. Yaji, T. Yamada, S. Kubo, K. Izui, S. Nishiwaki, A topology optimization method for a coupled thermal-fluid problem using level set boundary expressions, International Journal of Heat and Mass Transfer 81 (2015) 878-888.

[30] V. Subramaniam, T. Dbouk, J. L. Harion, Topology optimization of conjugate heat transfer systems: A competition between heat transfer enhancement and pressure drop reduction, International Journal of Heat and Fluid flow 75 (2019) 165-184.

[31] J. K. Guest, J. H. Prévost, Topology optimization of creeping fluid flows using a darcy-stokes finite element, International Journal for Numerical Methods in Engineering 66 (3) (2006) 461-484.

[32] C. McConnell, G. Pingen, Multi-layer, pseudo 3d thermal topology optimization of heat sinks, in: Proceedings of the ASME 2012 International Mechanical Engineering Congress, Houston, 2012.

[33] J. H. Haertel, K. Engelbrecht, B. S. Lazarov, O. Sigmund, Topology optimization of a pseudo 3d thermofluid heat sink model, International Journal of Heat and Mass Transfer 121 (2018) 1073-1088.

[34] S. Zeng, B. Kanargi, P. S. Lee, Experimental and numerical investigation of a mini channel forced air heat sink designed by topology optimization, International Journal of Heat and Mass Transfer 121 (2018) 663-679.

[35] T. Van Oevelen, M. Baelmans, Numerical topology optimization of heat sinks, in: Proceedings of the 15th International Heat Transfer Conference, Begell House Publishers; 50 North Street, Danbury, Connecticut 06810, 2014, pp. 10-15.

[36] P. Lacko, M. Blommaert, W. Dekeyser, M. Baelmans, Filtering in microchannel heat sink topology optimization, in: Proceedings of the 13th International Conference on Heat Transfer, Fluid Mechanics and Thermodynamics, Portoro, Slovenia, 2017, pp. 919-924.

[37] W. Kays, M. Crawford, Convective heat and mass transfer, 3rd Edition, Tata McGraw-Hill Education, 1993.

[38] T. J. Hughes, L. P. Franca, M. Balestra, A new finite element formulation for computational fluid dynamics: V. circumventing the babuška-brezzi condition: A stable petrov-galerkin formulation of the stokes problem accommodating equal-order interpolations, Computer Methods in Applied Mechanics and Engineering 59 (1) (1986) 85-99.

[39] A. N. Brooks, T. J. Hughes, Streamline upwind/petrov-galerkin formulations for convection dominated flows with particular emphasis on the incompressible navier-stokes equations, Computer methods in applied mechanics and engineering 32 (1-3) (1982) 199-259. 
[40] T. E. Tezduyar, S. Ramakrishnan, S. Sathe, Stabilized formulations for incompressible flows with thermal coupling, International Journal for Numerical Methods in Fluids 57 (9) (2008) 1189-1209.

[41] C. Le, J. Norato, T. Bruns, C. Ha, D. Tortorelli, Stress-based topology optimization for continua, Structural and Multidisciplinary Optimization 41 (4) (2010) 605-620.

[42] M. H. Y. Tan, A. R. Najafi, S. J. Pety, S. R. White, P. H. Geubelle, Gradient-based design of activelycooled microvascular composite panels, International Journal of Heat and Mass Transfer 103 (2016) $594-606$.

[43] B. Bourdin, Filters in topology optimization, International Journal for Numerical Methods in Engineering 50 (9) (2001) 2143-2158.

[44] T. E. Bruns, D. A. Tortorelli, Topology optimization of non-linear elastic structures and compliant mechanisms, Computer Methods in Applied Mechanics and Engineering 190 (26) (2001) 3443-3459.

[45] K. Svanberg, The method of moving asymptotesa new method for structural optimization, International journal for numerical methods in engineering 24 (2) (1987) 359-373.

[46] F. Okkels, H. Bruus, Scaling behavior of optimally structured catalytic microfluidic reactors, Physical Review E 75 (1) (2007) 016301.

[47] M. P. Bendsøe, O. Sigmund, Topology optimization: theory, methods, and applications, Springer, 2004 .

[48] J. P. Groen, O. Sigmund, Homogenization-based topology optimization for high-resolution manufacturable microstructures, International Journal for Numerical Methods in Engineering 113 (8) (2018) $1148-1163$.

[49] L. H. Olesen, F. Okkels, H. Bruus, A high-level programming-language implementation of topology optimization applied to steady-state navierstokes flow, International Journal for Numerical Methods in Engineering 65 (7) (2006) 975-1001.

[50] R. Shah, A. London, Chapter vi - parallel plates, in: R. Shah, A. London (Eds.), Laminar Flow Forced Convection in Ducts, Academic Press, 1978, pp. 153 - 195. 